\title{
Pathophysiology of Biophoton: Vibratory Impact Syndrome Leading to Physical Effects and Metabolic Changes-Part 1
}

\author{
Luiz Gonzaga Camelo \\ Taguatinga, Distrito Federal, Brazil \\ Email: luizgcamelo@gmail.com
}

How to cite this paper: Camelo, L.G. (2021) Pathophysiology of Biophoton: Vibratory Impact Syndrome Leading to Physical Effects and Metabolic Changes-Part 1. Neuroscience \& Medicine, 12, 126-162. https://doi.org/10.4236/nm.2021.124011

Received: November 1, 2021

Accepted: December 17, 2021

Published: December 20, 2021

Copyright (อ 2021 by author(s) and Scientific Research Publishing Inc. This work is licensed under the Creative Commons Attribution International License (CC BY 4.0).

http://creativecommons.org/licenses/by/4.0/

\section{(c) (i) Open Access}

\begin{abstract}
The emission of the ultra-weak photon or "biophotons" is a phenomenon resulting from the spontaneous emission of electromagnetic frequencies in the range of $240-650 \mathrm{~nm}$ [nanometers] absorbed by all living beings, including man. It is measurable in the UV region with a highly sensitive electromagnetic device. This is a case study of a 60 -year-old patient with pathological biophoton emission. I assume it is the first study reported in the literature. And the most surprising, occurred in the author himself. Therefore, I am the author and the patient reported in this study. Seven years ago, I was surprised by a large emission of "biophotons" starting in some places on the body and spreading throughout the body over a period of three months, which continues to this day. I follow up from the beginning, with periodic clinical and laboratory examinations without the need for hospitalization and consequent opening of medical records. Currently, biophoton emission is already wellcontrolled, perhaps with an emission around 5\%, although with different characteristics from the initial clinical picture, so I am already writing part-2 of this study. It appeared spontaneously and without the interference of any external factor. It follows an intelligent and intentional pattern. On the one hand, this study showed negative effects such as an increase in glucose, triglycerides and cholesterol; left leg sciatic nerve involvement and right saphenous vein injury. On the other hand, it also showed unusual effects, such as a significant increase in serum testosterone levels, delayed aging and increased libido. I would say an excessive increase in libido. In this article, we will address the Pathophysiology of biophotons and some aspects that are not in accordance with literature data through direct observation. This article describes the physiology and some aspects of biophoton pathology. We will also address a possible role of Consciousness in the emission of biophotons. Then we will present some hypotheses, speculations and research suggestions and
\end{abstract}


perspectives for the future. Finally, we will address "intriguing phenomena" of a strange syndrome using the direct observation method. For convenience, we're going to use the term "energy" a lot, which seems pretty familiar to me.

\section{Keywords}

Energy, Photons, Bio-Communication, Ultra-Weak Photon Emission, Biophotons, Consciousness

\section{Introduction}

Vibratory impact syndrome is an energy disorder due to the excessive and pathological emission of Biophotons. In fact, it is an energy emission perceived on the periphery of the body, after its condensation. It is of unknown cause leading to metabolic changes and multisystemic effects.

The studies of Quantum Mechanics in recent years have provided subsidies for a better understanding of energy and matter and the consequent influence of the mind. Everything in the universe is energy, movement, frequency, vibration and dynamics. Therefore, mass and energy are two aspects of the same reality, maintaining, however, the same equivalence represented by the equation $\mathrm{E}=$ $\mathrm{mc}^{2}$ that immortalized its creator, Albert Einstein.

Biophoton is an ultraviolet [UV] wave, of low vibrating frequency, invisible to the naked eye and visible only to highly sensitive electromagnetic devices. In other words, it is a very low intensity electromagnetic wave emitted and absorbed by all living beings in the $200-650 \mathrm{~nm}$ range, spontaneously and continuously [1] [2]. Living beings, in turn, are considered emitters and receivers of "electromagnetic frequencies" for their regulation and development of their structural constituents, such as cell differentiation and growth [1] [2].

It is important to note that in humans, the "photon", a high frequency light package is absorbed and converted into biophotons by the "Mind" or nonbiological brain [3]. It is worth mentioning that the "biological brain" is not intelligent and does not think. Intelligence and thought are attributes of Consciousness [3]. On the other hand, the biological brain, comparable to a highvoltage transformer, reduces the frequency of psychic waves that come from the immaterial mind or non-biological brain. Despite being a noble organ in its function, the brain is just a "tool stuff" that is dedicated to working on behalf of its faithful operator. It is seen as an electromagnetic field operated by a "hidden entity", an intelligent, multidimensional being that transforms the world [3].

In fact, biophoton is semi-material energy emitted by man and is variable in intensity. Undoubtedly, the intensity of the biophoton emission depends on the activity of cellular metabolism and specific anatomical distribution sites throughout the body [1] [2]. The greater this activity, the greater the production of biophotons to supply the demand for neuronal activity, energy metabolism, cerebral blood flow, as well as oxidative processes [1] [2] [4], which proves to be neces- 
sary energy for the maintenance of life.

Biophoton, therefore, should not be considered a harmful by-product of vital processes. This energetic flow descends vibratingly from the "non-physical mind", condenses and is thus used in the form of biophotons or vital energy. Therefore, all energy that circulates in the human body is of mental origin that accumulates in the vital body, the biological field of man [5] [6]. In fact, without the mind, there are no biological functions. Biophoton is, in the last analysis, a "psychophysical energy" that depends on the organic metabolism to be potentiated. It is necessary to understand that the human being is an integral, holistic being [5] [6], taking into account his energetic constitution. In this way, despite being linked to organic life, the human being is the only being that is simultaneously linked to other "electromagnetic fields" made up of ever more subtle energy [3] [5], such as the physical, energetic, emotional and mental fields.

\section{Role of Consciousness in the Emission of Biophotons}

Based on the physical body, the densest field and coherence properties that regulate the universe, it is reasonable to believe in other less dense, more fluid vibratory bodies. We assume that the chain of events that arises from the atom to the periphery of the physical body has a corresponding "fluid matrix" [3] [5]. An organizing biological field that involves and permeates living bodies [3] [7]. In this context, all the constituents of the human body form a "common electromagnetic field" vibratingly interconnected. In other words, each atom or molecule has its corresponding fluidic field and so on until it encompasses the entire body [3] [5] [7]. We are talking about a matrix or model, an "omnipresent fluidic body" [7] involved by Consciousness, the intelligent being that constitutes man, forming a "perennial" electromagnetic field responsible for the entire anatomo physiological constitution of the human body [3] [5] [7] [8]. We will call it "Psychosome" or self-replicating psychic body [3]. Here, there is no duality. It's just a word. In fact, it is the subjective or energetic body of the human being.

The Psychosome, therefore, is a fluidic [6], electromagnetic [7] body of high vibratory frequency surrounded by the divine spark [3] [8]. Called consciousness, the "divine spark", a vibrating matrix, where mental energy is generated [6] is a hidden and eternal "entity" [3]. It is, therefore, an intelligent, coherent and non-random electromagnetic field. In this way, the human body does not generate an electromagnetic field [3]. It just externalizes a "biofield" generated at another vibratory level that emanates from an electromagnetic matrix [3] [6] [7]. In fact, this energy comes from the non-biological brain, from the depths of the unconscious. This brain certainly has a "holographic copy" of all things [5], in a perennial way, including a subatomic particle [8]. It is in this brain that memory is stored [3].

Consciousness, therefore, has a nature similar to an information field [5] that can play a mediating role between underlying fields [7] and the human organism [3] [8] [9] [10]. It is important to remember that Consciousness instantly inte- 
racts with entangled states [8] [10] [11] [12] that may be responsible for active information between higher order processes and neuronal events, that is, active interaction of a physical and mental nature [5] [9] [10] [13] [14] [15]. In fact, a wide range of research suggests the notion of a coherent and self-replicating biological organizing model [3] that stores and processes higher order information [10] [12] [14].

Surprisingly, "human consciousness" [6] [14] is non-spatial and non-temporal [10] [15] [16] connected to Universal Consciousness [6] [8] [9] [15] [17] from which it takes life support [18], including photon. In fact, this new view is in line with Bell's theorem of non-lacality [7] [10] [19] and the principle of causality [10] [14] [16] in quantum physics. Thus, man is connected to any human being, in any space-time dimension, through a vibrational correspondence, that is, an electromagnetic resonance [5] [10] [12] [19].

The mind is the fluidic, immaterial brain governed by Consciousness [5] [15], the Intelligent Being of the universe [3]. In this sense, the mind is the "perennial" [15] [20], holographic matrix of the biological brain through which Consciousness communicates with the environment [3] [5] [8]. In other words, Consciousness is an intelligent and omnipresent matrix [3] [15] which forms a morphogenetic field [3] [7] [8] [10] [12]. Here, matter does not produce Consciousness. It's impossible. On the contrary, it is Consciousness that acts on matter [3]. In fact, Consciousness does what it wants with matter. It is noteworthy that Consciousness is sovereign and absolute [3]. In this context, Consciousness, mind and biological brain interact with a coherent and organizing “electromagnetic field” [12] [14] which we call Psychosome [3] [5] [7].

From that perspective, it is from the non-biological brain [mind] [3] [5] governed by Consciousness [3] that the order or bioinformation comes for a simple tissue healing [6] [7] [10] [12] [14]. For example, who regulates cell division and differentiation? Without a doubt, it has to be an intelligent electromagnetic field [3], a holographic memory [8] [10] [12] [19]. Here, electromagnetic waves and fields can be the basis of biological organization [3] [7] [10] [12]. A principle is so simple that it can be found in coherence [5] [7] [9] [12] [19]. In fact, we cannot rule out the possibility of man having other vibratory or electromagnetic bodies [6] [7] [8] [9], vehicles of manifestation of the Intelligent Being [3].

As everything in the universe is coherent [5] [8] [9] [12] [14], by analogy to the physical body, the "Psychosome" or perennial fluidic body, semi-material is vibratingly coupled to other fluidic bodies [6] [7] [8] through which electromagnetic energies circulate, from of the non-biological brain [3]. Here, the biological brain transforms quantum events into classical events [12] [13] because it is coupled to the ubiquitous intelligent fluid brain [3]. In this context, we agree with the quotation by Meijer [21], when they say: our brains can operate as a holographic frequency analyzer, decoding projections of an underlying, more fundamental dimension. There is, therefore, a type of coupling of different electromagnetic fields [3]. Thus, we find a kind of characteristic "vibration signa- 
ture". In other words, each atom, molecule, substance, cell, organ, system and entire body has a matrix that forms a fluidic signature [3] [6] [7] [8] [10]. Here, there is a summation sequence of fields to form a common electromagnetic field, the Psychosome [3]. From the Psychosome, mental energy radiates until it reaches the "vital body" or energy body where all the energy "quanta" of the human being accumulates. From this vibratory level, slightly more subtle [6] [7] than the physical body, this energy reaches all the cells and organs of the body, in the form of vital energy, where it will be metabolized and, in this way, eliminated by natural pathways.

\section{Origin and Destination of Biophotons: The Hypothesis}

Photon, like oxygen, is not synthesized in the laboratory or produced by living beings, including man. It has been ubiquitous in nature since the Big Bang. In fact, photon excites electrons inside atoms which, in turn, produce energy through their nuclear elements. Excited electrons can revert to more stable energy levels and then emit photons leading to electronic bonds between molecules. Finally, the breaking of such bonds can, by a reverse process, again lead to electron excitation and the process continues.

The cell, the basic unit of biological life, not only externalizes a biofield [10] but also absorbs and emits biophotons [1] [2] [18]. Based on bioinformation [10] [18], cells maintain communication with each other and with the being that gave rise to them, even being in cell culture [10], as well as communicating with the "Universal Consciousness" [6] [8] [22], the Supreme Intelligence of the Universe. Therefore, we are interconnected, holographically, forming an "infinite web", an entanglement [10] [11] [14] [18] according to the new concepts of quantum field mechanics. There is not a single particle that is not radiated by Universal Consciousness [3]. We are part of that Consciousness that never dies!

\section{Nucleic Acid: DNA}

Probably these ref. [1] [20] [21] [22] [23] [24], when inserted in the "template", they were subtracted.

We assume that the DNA, holographic information field [6] [8] is the interface between several electromagnetic fields [10] [12] [14]. Here, the main role of DNA is to store information [8] [14]. Thus, the photon, subtle energy, is converted into biophotons, low-frequency energy, and in this way, it is transported, saturated with information [8], to the neuronal mitochondria, where, in fact, the conversion takes place through metabolic processes [1] [20]-[25]. In this context, the metabolic processes that produce biophotons inside the mitochondria supposedly lead to the production of ATP. These processes are managed by the interaction of a radiation field coupled to biological systems [12], called Psychosome, an omnipresent morphogenetic field [3]. From this vibratory level, biophoton is distributed to all organs and systems [23]. Therefore, biophoton is an essential energy for life [18], just like oxygen. In fact, we presume to be the vital 
energy used in "healing" by healers [10] [11] [14]. It is the energy that goes into the composition of the "Bioenergy" of the healers, receiving their own healing qualities [7] [10] [11] [18] [26]. Without a doubt, Consciousness is the fundamental cause of our health or our illness [6] [12].

It is important to highlight that DNA, the essence of biological life, somehow exhibits consciousness [6], but it is not intelligent [3]. It is identified as a biological laser [5] [6] [8] [12] [27]. Therefore, a coherent "non-local" field that denotes the origin and destination of biophotons [21] [27]. No doubt, DNA functions as an antenna [14] capable of encoding and decoding holographic information [6] [12] [28]. In fact, it is not just a molecule made up of an original sequence of bases that forms an electromagnetic field [5] [6]. It is also a store of photons [22]. In this context, it is governed by an Intelligent Being [3] who supports it and allows its unconditional replication.

On the other hand, the Psychosome, a self-replicating psychic body [3] condenses the "photon" coming from the immaterial mind and interacts with the DNA [27] to form a "radiation field" coupled to biological systems [10] [12]. Undoubtedly, a morphogenetic field with regenerative properties [12] [14] in perfect synchronization with Consciousness [15] and the consequent emission of biophotons [1] [2] [18]. Biophotons, therefore, are highly coherent fields generated in the DNA that become the organism's main communication network and the most fundamental regulatory source of organic life processes. In this context, it regulates not only growth, cell differentiation and regeneration, but mainly the entire morphogenesis of living beings [27]. Undoubtedly, we believe that "Consciousness" [3] plays a fundamental role in this process [6] [8] [10] [11]. I don't mean biological consciousness, if it exists at all. I refer to the immaterial, omnipresent Consciousness, the Higher Self [3]. It uses the endogenous medium [6] to transport biophotons possibly through the mitochondria.

The mitochondria, "energy converter plant", are separated from the energetic or vital body by an almost insignificant vibratory barrier. In this context, it is important to emphasize that, in cells; mitochondria are a biological deposit of DNA, as well as the main source of biophoton emission [28]. Therefore, mitochondria are not static inside cells and, thus, constitute "dynamic networks", leading to changes in shape and molecular exchange in the short term [28]. In this sense, when transported and distributed, the "biophoton" plays its role as vital energy, that is, its use in healing therapies, protein synthesis, digestion and removal of waste products [29] [30]. In this way, it is emitted, without loss of its vitality, and is absorbed by other living beings [1] [2], and the cycle continues ad-infinitum. Just like oxygen, biophoton, physiologically will always be biophoton!

At all times and in all cultures there have been reports of healings attributed to subtle energy [7] [10] [18]. We believe that biophoton is this energy [18], even more subtle than electromagnetism, not identified by science and that may be involved in healing [7] [10] [14]. In fact, this energy emanates from a hidden 
"Consciousness" that controls the conscious intention [18]. It is important to emphasize that Consciousness is not the same thing as free will. Free will is one of the attributes of Consciousness. Therefore, free will is the ability to consciously decide [31]. Without a doubt, the biophoton is the alloy, the glue that rebuilds tissue and heals wounds [7] [10] [14]. In this context, there is an unconventional energy field emitted by a "perennial matrix" from our inner world. Therefore, biophoton is a natural restorer [10] [14], that is, anti-inflammatory, analgesic, bactericidal, healing, as well as an essential biological regulator.

It is possible that the "retina" works as a "biological device" that reduces vibrations, which, in turn, transforms external photon signals into electrical signals and not just any organ that can generate or absorb frequencies [4] [17]. In fact, when the retina captures the photon, it reduces its frequency and transports it to the brain [20]. Therefore, all living cells, including neuronal cells, animal, plant and human cells that contain mitochondria and DNA emit biophotons [4]. In this case, the brain, seen as a coherent system in the biophysical and quantum biology context, is not a photon producer, but only a converter and distributor [17] [20] [28].

It is noteworthy that deluded science, on the one hand, gets lost in costly scientific brooding, forgetting the fluidic and definitive nature of the human being. The researcher, on the other hand, before looking for the answers to his intellectual cogitations in the external world, looks for them in the inner world.

For the purpose of differential diagnosis, some conditions such as diabetes, dyslipidemias, peripheral neuropathy, dermatomyositis, avitaminosis B12, superficial mycoses and ectoparasites were excluded.

\section{Results}

It all started a week after my wife's breast cancer removal in January 2014. Here also began my ordeal. While researchers were burning biophotons in the cold of labs, I was revealing to myself "living evidence" of events related to the pathological emission of biophotons.

I am inside an experimental laboratory that is myself. Without a doubt, I have experienced everything that is written in this clinical trial. It's very painful! I thought of something unspeakable. It's very weird! One day, I was walking in a park and I had the perception of some micro perforations in my legs, from the inside out, exteriorizing as if they were "electric sparks" with the perception of slight itching or shock. The most objective perception possible was when "sparks" appeared in the face, something similar to "lightning" in a stormy night. Thereafter, the events gradually increased, reaching thousands in a short time, with greater intensity, in the entire extension of the lower limbs to the tips of the fingers. Then the "rays" are directed to feet with thousands of micro perforations protruding about $2 \mathrm{~cm}$ beyond the fingertips. And finally, there was dissemination to some parts of the body, until they lodged in the arms, reaching the hands from where an enormous amount of energy was emitted that pro- 
jected beyond $2 \mathrm{~cm}$, from the fingertips.

By about three months, the elimination of energy was immeasurable, across the entire body surface with billions of microperforations. I wanted to go crazy, several times, at that time. The most concrete perception was of a "cylinder" of gas with billions of microperforations. Energy was eliminated from every pore. Here, perceptions were of the needle piercing type, sometimes very strong. All the hair on the body and the eyelashes and ear canal were saturated with energy, especially in the right eye, where the eyelashes were also twisted and somewhat stiff as if I were using beauty products on my eyelashes. The perception in the eyes was of sand or any other foreign body, which remained for 24 hours, being eliminated when the eyes were washed with saline solution of sodium chloride or natural water. During this period, a surprising fact emerged. I was lying with my hands on my chest when I heard a muffled "click" coming from my fingers: then, I thought, I broke a finger! When I looked down at my hands, I saw that I had only broken my wedding ring, the way you break a strand of hair. That simple! I was panicked. He could break bones, I thought! Until then, I didn't know anything about this intriguing disease. Disease? Perhaps mind sickness!

Undoubtedly, the emission of biophotons follows a changing daily pattern. At dawn, around 5:00 am, I would wake up lightly, thinking that everything would disappear in the next hour. Sheer mistake! It was just a matter of moving some muscles and the emission of energy began, which increased more, until it stabilized at the maximum peak. This increase was quite a bit in the afternoon and mainly in the late afternoon and even more during the night. I could even time it with British accuracy. In fact, the symptoms worsened considerably from 5:00 pm to 5:00 am. The emission of biophotons follows a pattern that varies in intensity, in all segments of the body. Thus, by sequence, the highest emissions occurred in the hands, feet, face, upper $1 / 3$ of the chest and neck, and finally in the genitalia.

The asymmetry of the biophoton emission was unquestionable. From top to bottom, it covers the entire right side of the body, that is, the auricle, eyes, nostril, hands and legs, leading to a lesion in the right saphenous vein.

In all these segments, the emission of biophotons was intense and permanent. I'm left-handed. I was intrigued by this fact, since in the literature there are more frequent reports of biophoton emission on the left. Until I found four individuals who also had this syndrome. All four are right-handed, whose Biophotons emission occurred more intensely on the left side of the body. Here, I see no competition between the cerebral hemispheres. It is possible that there is only a "cognitive" correlation with predominance in one or the other hemisphere. Today, I follow up on the four cases.

\subsection{Change in Body Temperature}

One night, in midsummer, in a tropical country, I was awakened with a strange 
cold. I presented glacial cold with tremors, without improvement, even with a blanket, despite the summer. I checked the temperature thinking I had hypothermia. The temperature was normal and the cold was only in the upper and lower limbs. Then, in five to ten minutes, I developed a torrid, desert temperature in my limbs. Here, in both cases, the improvement was instantaneous when I submerged my legs or arms in a basin of water or in the hydro-massage bathtub, see treatment section. After a few more minutes, everything disappeared. From this episode, I learned that every time I "cooled" the limbs, it was because of retention and accumulation of energy and when it "warmed up" it was to release energy from the limbs, normalizing the temperature.

\subsection{Biophoton Emission Phases}

The pathological emission of biophotons is characterized by having three phases:

1st. Acceleration phase or excessive emission;

2nd. Deceleration or energy retention phase;

3rd. Excessive energy loss phase.

The acceleration phase was characterized by excessive biophoton emission. It was dramatic. Here, the pain in leg muscles was mild, deep, intuitive and continuous. The main symptom was irritability. It was as if the brain was saturated with energy. I felt like going crazy. At this stage, I met a patient who spent three months in the asylum. Duration of this phase: 2 years or more.

The deceleration phase was characterized by energy retention or accumulation. Due to the retention of energy, I did not feel "hungry", I had the perception of satiety and even so there was a weight gain of around $4 \mathrm{~kg}$. In a seven-year period of evolution of this syndrome, in addition to weight gain, there was a slowdown in aging. It was like I had an overfeed. This phase starts by suppressing the energy emission from the place that emits fewer biophotons: anterior and posterior face of the trunk. Then, the deceleration reached other segments until it lodged in the hands and feet. Finally, poor digestion was also noted for several days, even of fruit, with accumulation of hot gas in the stomach. It is the worst of all phases, with a very long duration. This phase remained in the last two segments [hands and feet] until the balance phase of the process. In fact, it never comes to an end. There needs to be energy balance all the time.

The excessive energy loss phase is due to the imbalance of the deceleration phase. It occurred due to the use of drugs under test or exposure to water, especially in the bath. Here, I had leg pain that specifically characterized this phase. See more on the pain topic.

\subsection{Beneficial or Therapeutic Effects of Biophoton}

It is known that water is a universal conductor of electricity. At the beginning of this "phenomenon", I noticed a profound relief after showering or washing my hands. I started by submerging my hands in a basin of water at room temperature or preferably cold. 
In the pool area, I looked at a plant in a jar that wasn't in good health. So I decided to take the water from the basin I was using and spray the sick plant. I repeated this procedure twice a day for three days. The plant came out of the near-death state to an exuberant life. I was excited and decided to stay submerged in a bathtub or swimming pool for 30 minutes. It was the best thing I ever did. I left the bathtub almost good, with little energy output. I took a bucket of water and sprayed the plants in the garden. It wasn't watering plants. The garden was beautiful! I concluded that from my body and especially my hands, a vital fluid essential to life was eliminated.

During this same period, my wife started radiotherapy treatment for breast cancer. She felt a lot of pain from the burning where irradiation was applied. At that time I was already a "cylinder" of punctured gas. I didn't even need to concentrate. I started to lay my hands on the chest region, the radiation site. Here, the emission of biophotons, under the intentional influence, could therapeutically affect the patient's health status, interacting with her biofield, leading to positive effects on her psychophysical balance. In fact, in less than five minutes, the relief was almost $100 \%$. I repeated this procedure several times a day, until the radiotherapy sessions ended. What a relief for both! Without a doubt, it is unequivocal proof that biophoton is absorbed by plants and other beings, as well as man. In all cultures and medical traditions, healing has been achieved by moving energy [6] [7].

\subsection{Main Biophoton Excretion Pathways}

The main natural, perceptible pathways of great energy elimination are: eyes, nose, mouth, hands, feet, genitalia, urinary and digestive tracts.

The elimination of biophotons from the oral and nasal mucosa was unprecedented. In fact, it left the oral/nasal mucosa extremely dry, in addition to causing many lesions.

The digestive tract is the main elimination pathway for biophotons. It is done through belching and mainly flatus. It does not appear to be "methane gas", whose odor is unmistakable. Here, there is no odor. It is noteworthy that this gas was not due to food digestion. The efficiency of the digestive tract is frightening. Here, biophoton can be considered a by-product of cellular metabolism. It was eliminated like all other by-products or slag eliminated in urine or feces.

In the biophoton retention phase, there is an unbearable accumulation of "hot gas" in the stomach, which is eliminated only with the ingestion of an extremely cold carbonated drink.

Urinary elimination is dramatic. It appears that large concentrations of biophotons in urine cause urethra spasm. The spasm is so violent that it obstructs the passage of urine and so when the duct opens the urine is excreted. Elimination is so fast it doesn't wait. It appears to be involuntary. I believe it is due to the accumulation of biophotons, rather than peripheral nerve involvement. The pain is intense until the sphincter relaxes. It looks like the lining of the urethra is be- 
ing pulled inside out. It should be noted that this pain is not in the flesh, in matter. There is a considerable improvement with the intake of several liters of water per day, perhaps by dilution of the urine and less concentration of energy. It continues to this day.

\subsection{Biophoton Dissemination Pathways}

The hematogenous system is the main pathway for dissemination of biophotons to cells. The action of biophotons on blood vessels is significant. There is dilatation of blood vessels with significant venous stasis, in lower and upper limbs. In the upper $1 / 3$ of the chest, neck and face, only vasodilatation occurs. Vasodilatation may be due to peripheral nerve involvement. A considerable portion of biophotons is also disseminated by the peripheral nervous system, altered electroneuromyography.

\subsection{Biophotons and Immune Status}

There appears to be some correlation of biophotons with the immune system. Until the year 2014 I had common virus, like cold very often. However, with the excessive emission of biophoton over seven years, I no longer had a common cold, except at the end of the process, when there was already a significant reduction in energy emission. This reduction was most significant with the use of a certain "test drug" used to accelerate the excretion of biophotons in the retention phase in order to reduce pain [see treatment section]. One day, suddenly, the "virus" appeared with great intensity. So, I suspended the drug that was being used, when I noticed again an increase in the emission of biophotons, which is immediately noticeable, and a consequent significant improvement in the virus in just 48 hours.

On the other hand, there also seems to be a positive correlation with bacterial infection. At the beginning of the picture, due to the pathological action of biophotons, I had many skin lesions, lesions between the toes with exposure of muscle tendons, deep muscle lesion in the right calf muscle and lesion in the 5th toe of the right foot. This episode occurred four times. I didn't feel pain, hemorrhage, or signs of bacterial infection, nor did I use antibiotics. Everything evolved to a cure, without complications and even at that time I worked in an "infectious" emergency sector in a public hospital.

\subsection{Main Places of Emission and Action of Biophotons}

Here, we are going to divide the human body into three places: head, torso and limbs.

\subsubsection{Head}

The scalp, probably because it is a delicate place, the measurement of biophoton emission is impaired. Here, the most important symptom was the burn in the right auricle (see Figure 1). Undoubtedly, there is a large amount of biophoton emission from the head through the scalp and hair, which is perceived in the 


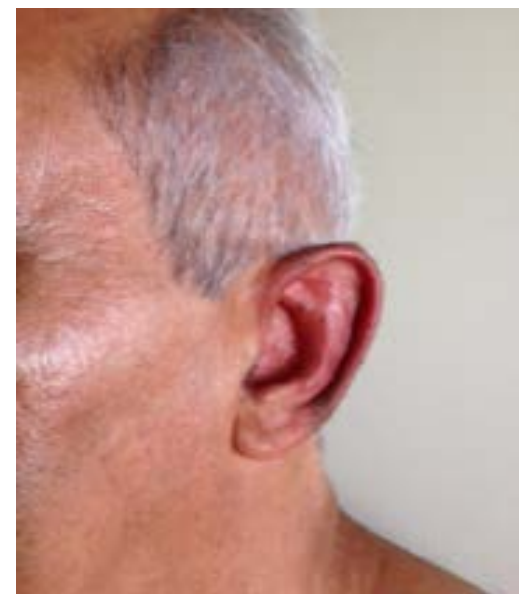

Figure 1. There is significant vasodilatation in the right auricle, beginning of the skin burning process in the right auricle. There was a $50 \%$ improvement after a long bath. It was at the beginning of the clinical picture, when I still didn't know anything about this strange phenomenon. I was afraid of losing the auricle!

form of very itchy microshock. There is also significant emission of biophotons from the ears. There is no correlation with literature data.

\subsubsection{Face}

All over the "face" there was emission of biophotons in the form of microshock or electrical sparks and itching with unmistakable perception. However, a large concentration of biophotons was located in the eyes, nose and mouth.

\subsubsection{Eyes}

The eyes, as they are very sensitive organs, are the place where the worst of all symptoms occurs. Here, the emission of biophotons is unbearable. Blurry vision. It is continuous and more frequent in the right eye, with perception of a foreign body. It's like sand in the eyes. When the condensation of this energy is very strong, white cords appear in the eyes with the action of a real foreign body. This still happens today. When the condensation of this energy is very strong, white cords appear in the eyes with the action of a real foreign body. This still happens today. I no longer have the Crystalline removed in surgery five years prior to this process. It works as a natural light reflector. In the patients mentioned above, the emission of biophotons through the eyes was similar.

\subsubsection{Nose}

In the nose, there was daily bleeding, more frequent in the right nostril, for more than three years. There was also elimination of fragments of nasal mucosa, such as devitalized tissue, with some frequency. Here, the worst symptom is the absence of mucus or mucosal lubrication and daily nasal obstruction, especially in the right nostril, until today. It is probably due to vasodilatation, aggravated by mucosal dryness. It is the second most unbearable symptom. As the end of the process approached, the deceleration of biophoton emission approached zero and the energy retention skyrocketed. Here, the nasal obstruction reached a crit- 
ical point that was no longer relieved with a vasoconstrictor-based solution. From the beginning, I use $0.9 \%$ sodium chloride solution, fifty-fifty with vasoconstrictor, Naphazoline hydrochloride at $0.5 \mathrm{mg} / \mathrm{ml}$.

\subsubsection{Mouth}

In the mouth, there was a change in the perception of flavors. The most striking flavor was bitter, followed by ferruginous. The salty taste was unbearable. My diet remained salt-free for thirty days, very frequent mouth dryness. It appears that biophoton disintegrates saliva. I thought it was dehydration; tongue burn, like hot milk burn; bleeding on the tongue, gums, cheek and lingual face of the lips. Also, there was disintegration of several dental restorations; some even more than once.

There was pseudoparalysis in the right cheek, leading to mucosal prolapse with frequent "bite" as if under anesthesia. I assume it's due to peripheral nerve involvement. Ulcers were on tongue, cheeks and lips, until today. Mild pain in all these lesions and healing in a few days, spontaneously or with the use of bee honey. Remarkable difference when compared to bacterial or viral lesions. These lesions were recurrent by the same factors several times over five years.

\subsubsection{Trunk [Torso]}

Here, the perception was not so concrete, despite the emission of biophotons on both the anterior and posterior sides of the chest. However, vasodilatation appeared in the upper $1 / 3$ of the chest and neck, also covering the right shoulder, with some frequency.

\subsubsection{Upper Limbs}

In the upper limbs, especially the hands, without a doubt, there is a greater emission of biophotons in the human body (see Figure 2). The projection of electric sparks, like "lightning", goes far beyond the extremities (see Figure 3 and Figure 4). Emission is continuous. The elimination is so intense that there was a kind of cooking of the tissue under the nails that covered the entire fingertips (see Figure 5 and Figure 6). Nails grew very often. Significant dryness of palms with loss of grip. The strangest perception was "dirty hands" or "oily hands" when wet, indicating involvement of peripheral nerves. There is significant vasodilatation and venous stasis (see Figure 7 and Figure 8).

There is great right-left asymmetry with very intense emission in the right hand. Undoubtedly, on the ventral side, the emission is more significant than on the dorsal side. It is noteworthy that the palmar or ventral region is densely vascularized. Elimination covers the entire length of the palm, with greater intensity on the digital extremities (see Figures 3-5). Here, I assume that vascularization is the main cause of increased biophoton emission that occurs in the human body (see Figure 7 and Figure 8). Elimination is also significant in the forearm, with burns on the inner face, with some frequency. Bone pain in the medial or internal epicondyle of the right humerus lasts four months. There was also pain in the medial or internal epicondyle of the left humerus for a short time. 


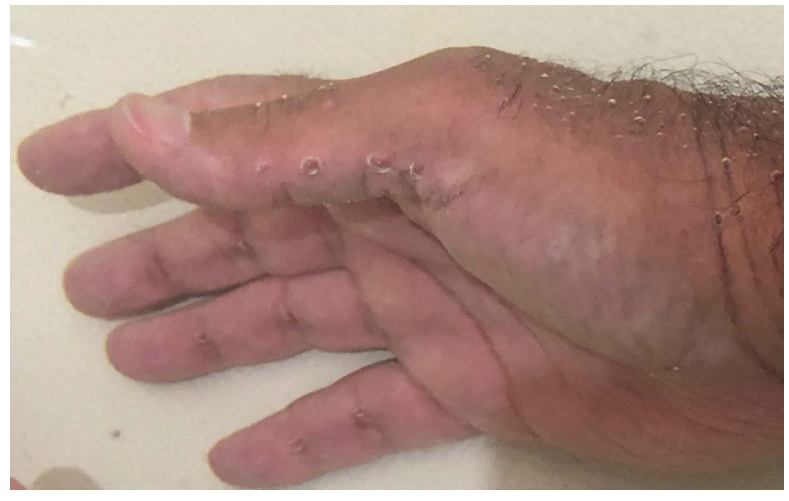

Figure 2. This is the physical representation of biophoton emission. Image captured at the bottom of a bathtub. Each bubble represents a flow of energy. At the beginning of the process, in each microspace, only bubbles were seen. The appearance of bubbles on the right palm at the end of the process is still surprising. There is significant venous stasis and bubbles on the palm and back. There is the perception of an electric spark being eliminated in the water that disappears in 3 minutes along with the bubble. What a relief!

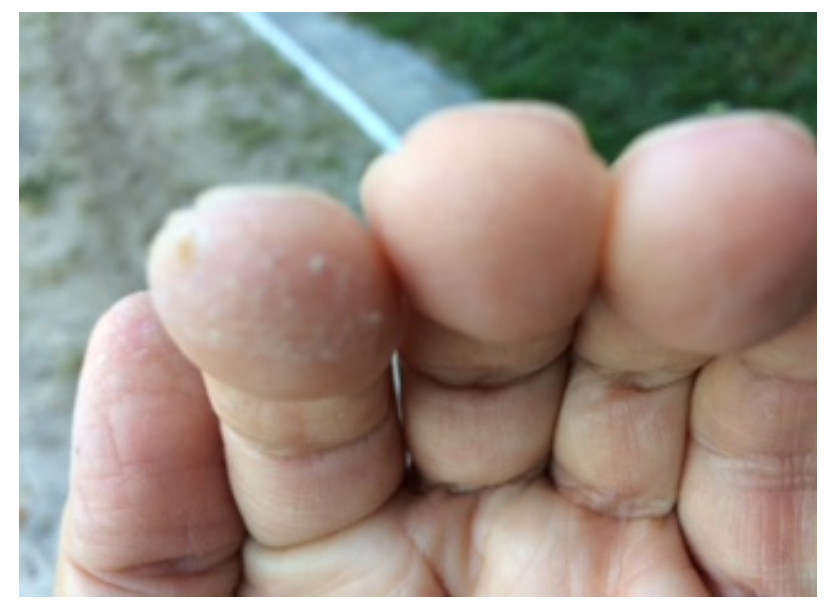

Figure 3. Note the pallor of digital extremities with some lesions, especially on the index finger and left thumb.

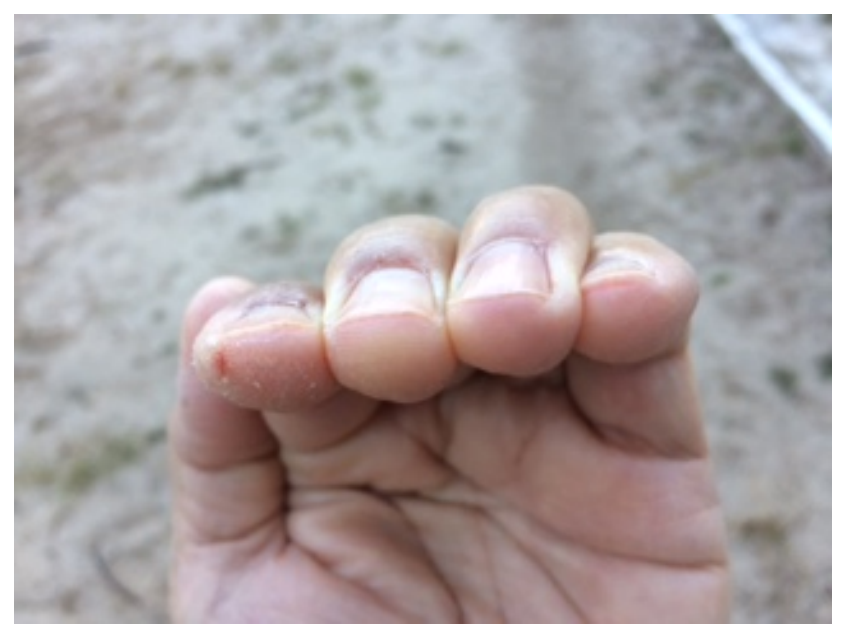

Figure 4. Here, the lesions are of the tissue displacement type under the nails with significant damage to the left index finger. 


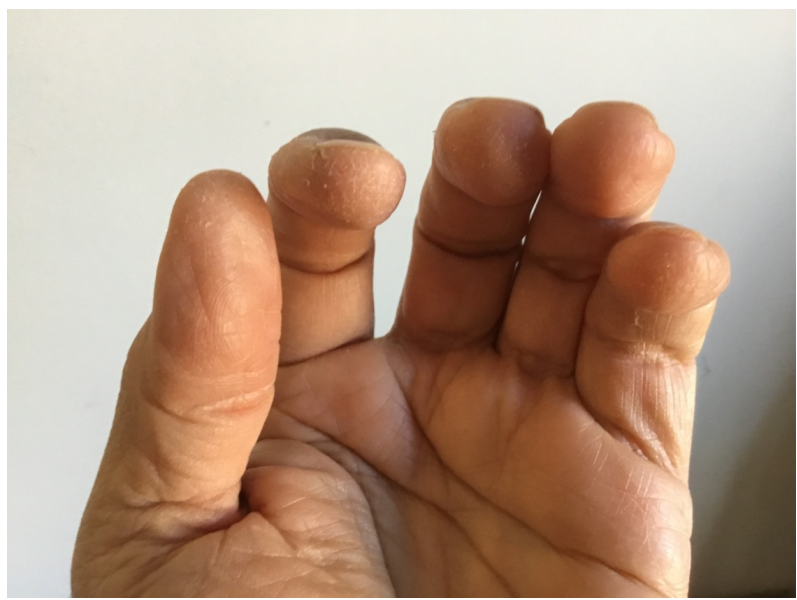

Figure 5. There is a lesion on the tips of the fingers of the left hand after 7 years of evolution.

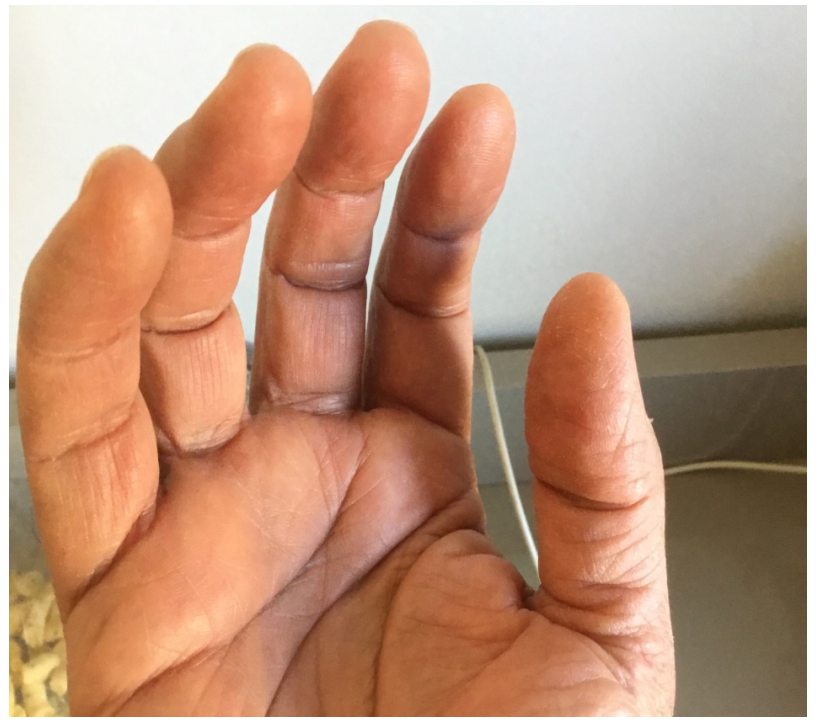

Figure 6. A lesion on the fingertips of the right hand is noted after seven years of evolution. It was the most committed hand in the beginning of the clinical picture.

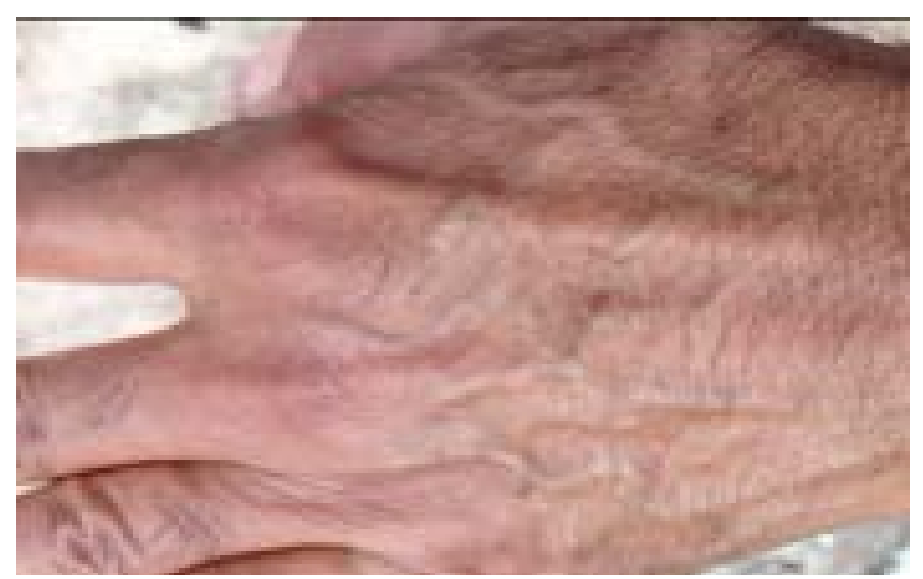

Figure 7. Vasodilatation and significant venous stasis, with tortuous and engorged veins, and painful sensitivity in left hand veins were observed. 


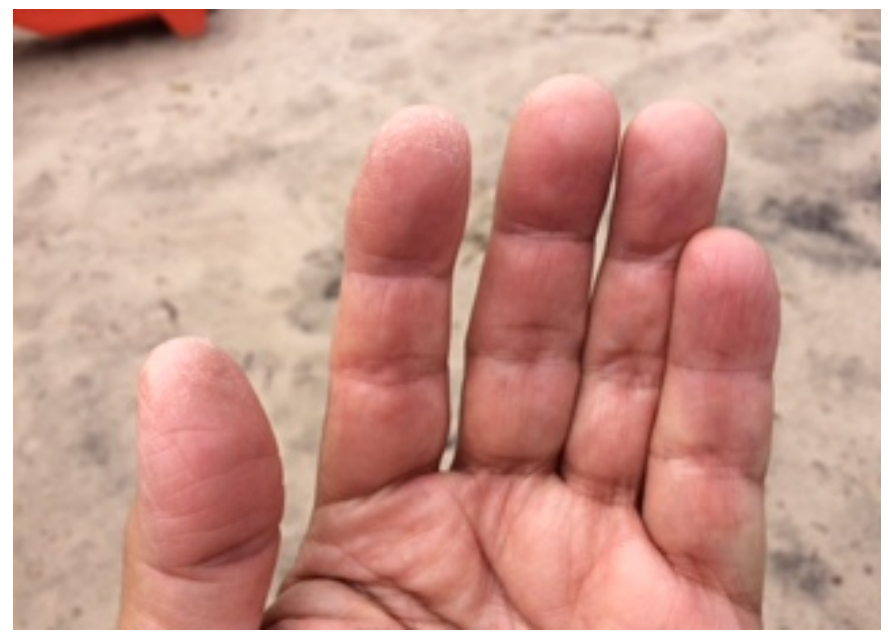

Figure 8. There is venous stasis in the left palm with pallor and lesions in the digital extremities.

\subsubsection{Lower Members}

Disabling and significant neuralgic pain on the anterior side of the right thigh lasting for six months. Also pain with "sciatica crisis" in the left leg due to sciatic nerve involvement since the onset of the condition. The appearance of muscle hernia was surprising. Three hernias with a diameter of $2 \mathrm{~cm}$ appeared in the right calf muscle and two in the left calf. The lower limbs are the second place where the emission of biophotons occurs with more intensity. It is noticeable throughout the length of the legs, ankles and especially feet (see Figure 9, Figure 10). The emission is more significant on the ventral or plantar surface of the feet. Here, plantar tissue dryness is notable with significant loss of adhesion.

The biophoton emission was so intense that it dissolved the "wax" on the floor, wherever I went. The emission is much more intense on the right foot. Here, the fifth fingernail [thumb] dropped four times (see Figure 10). Absolute absence of pain or bacterial infection. There is a nail lesion in the right foot; deep fissures in the calcaneus, as well as in the lower third of the leg with a papule, deep lesion in the calf. There was also a lesion between the fingers with exposure of muscle tendons, cadaver-like tissue, and a burn in the lower 1/3 of the leg (see Figures 11-17).

\subsection{Influence of External Light on Biophoton Emission}

So far, I didn't know of any "bizarre" effects of sunlight on the emission of biophotons. One day, I went for a walk for about 10 minutes under a cloudy sky at around 5:00 pm. Suddenly, the sun appeared in front of me huge and bright. At that moment, there was certainly triple the instantaneous emission of biophotons. I panicked, thinking I was on fire. It was a warming coming from the depths to the periphery, instantly. Here, it appears that the "solar photons" have entered an excitation process. In this way, they acted on the body's electrons, raising their energy power, emitting more biophotons. The external influence of sunlight was notable. From that day on, I avoided walking in the sun for several 


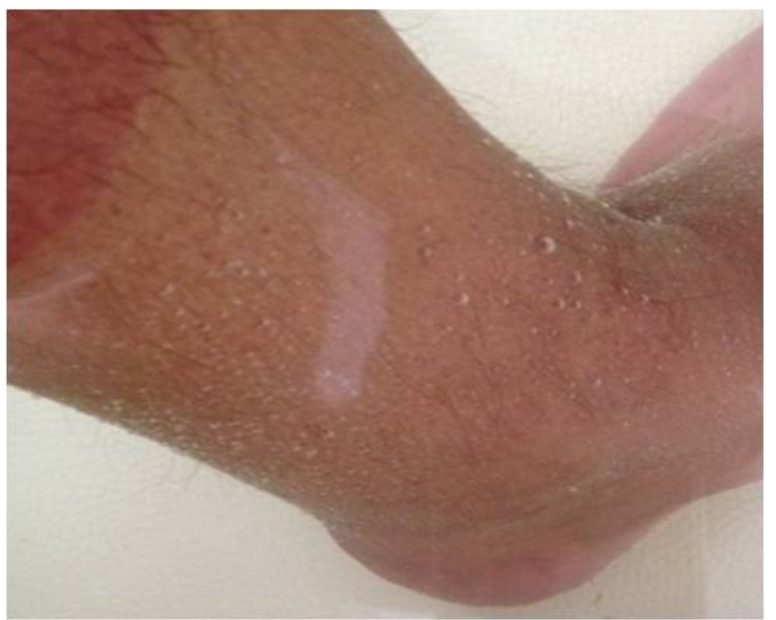

Figure 9. Emission of biophotons in the form of bubbles, whose image of the lower $1 / 3$ of the left leg was captured from the bottom of a bathtub. Normal people don't emit energy this way. This is a pathological emission of biophotons.

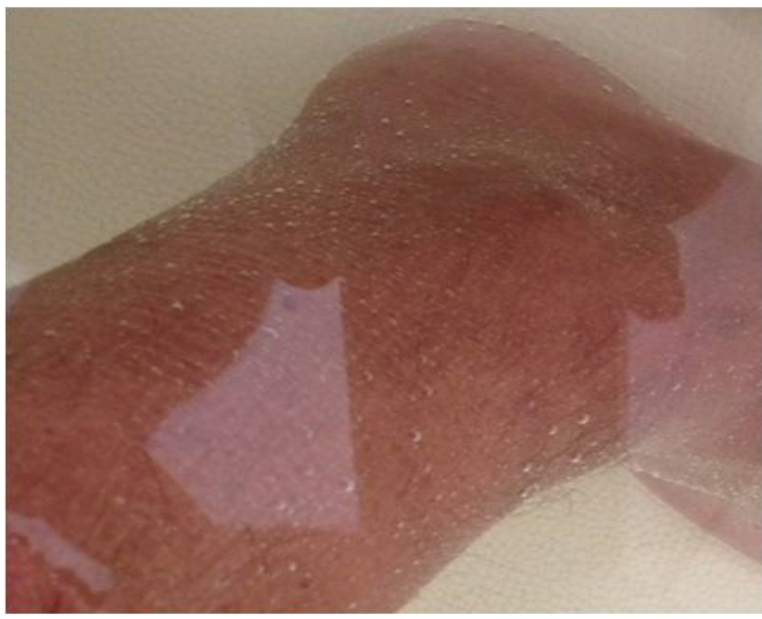

Figure 10. Emission of biophotons in an image of the lower $1 / 3$ of the right leg. We noticed few reflections of light in this image.

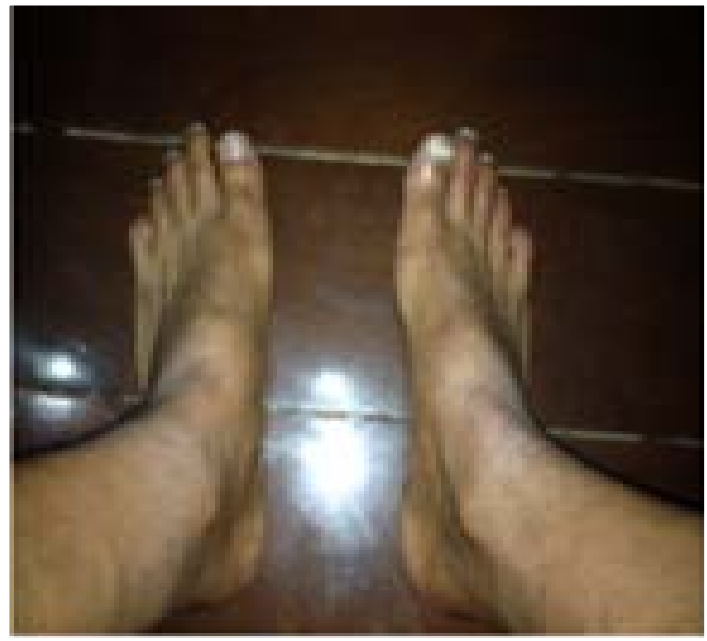

Figure 11. Skin burn in lower $1 / 3$ of legs and feet with lesion on the right toenail. 


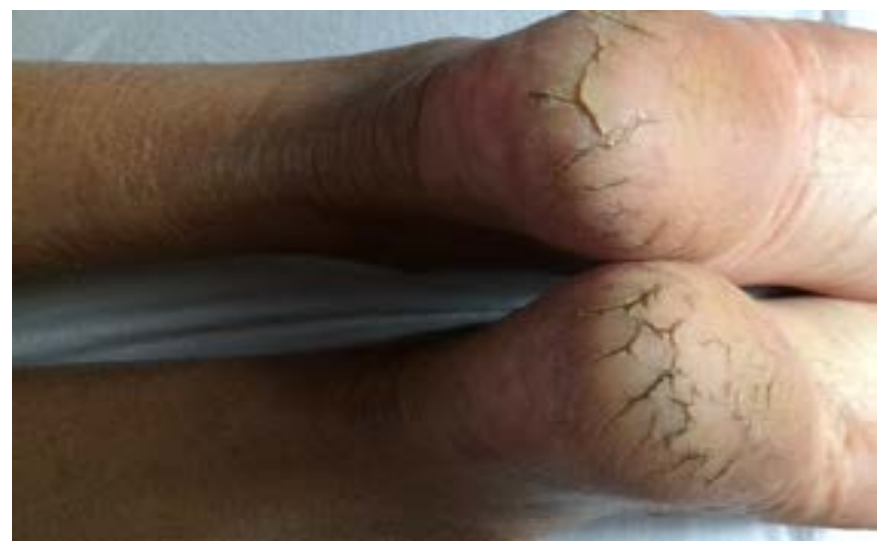

Figure 12. Note deep fissures in the heels as well as burns in the lower $1 / 3$ of the legs.

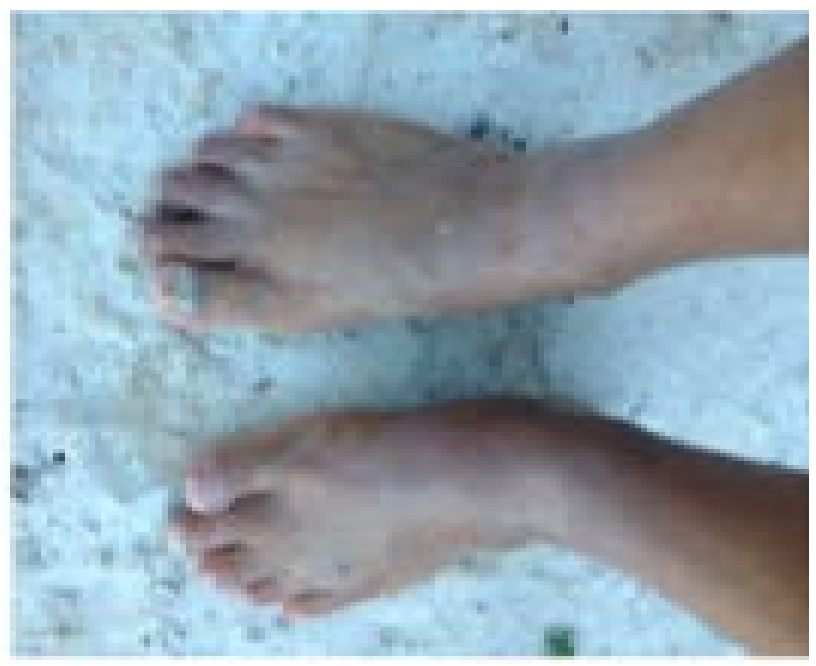

Figure 13. Note cutaneous burn on the lower $1 / 3$ of the legs and feet with a punctiform lesion on the dorsum of the left foot. Right nail injury.

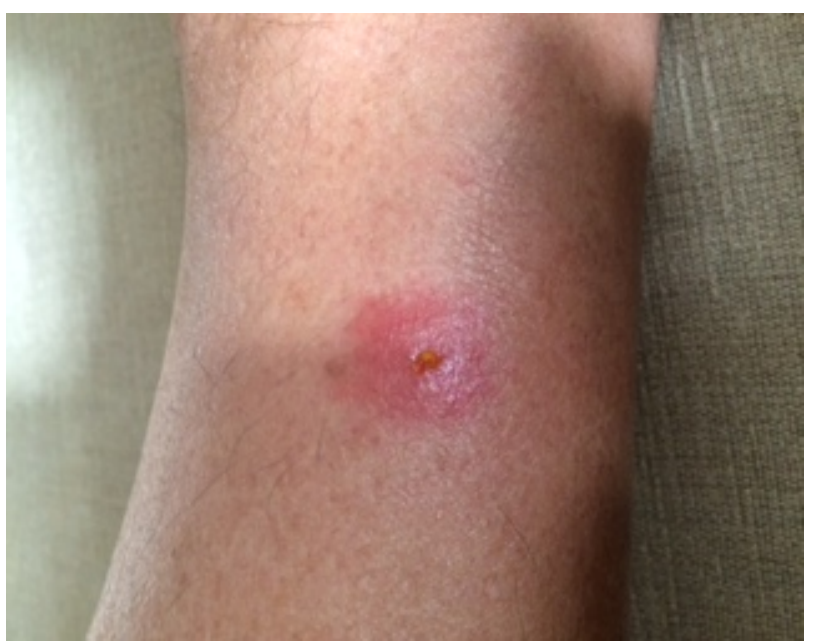

Figure 14. Note a cutaneous papule with erythema and a punctiform lesion infiltrated by biophotons, with extremely pruritic plasma drainage in the middle $1 / 3$ of the right leg. It always appears in lower or upper limbs, in number of one or two lesions. Recurrent injury. 


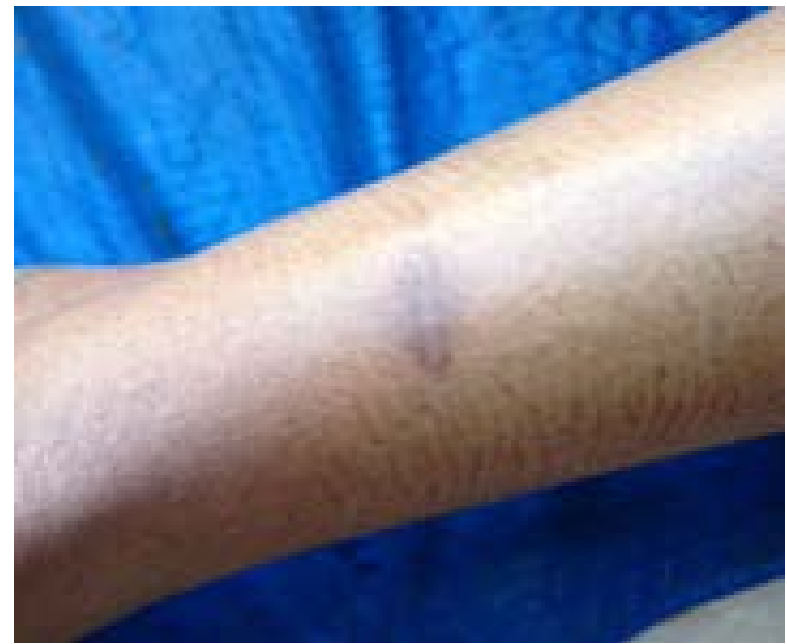

Figure 15. There is a scar due to a deep, cutting-type lesion in the right calf. I didn't go to the hospital for sutures because the doctor wouldn't understand the reason for the injury. No bleeding, no pain and perfect healing in a few days.

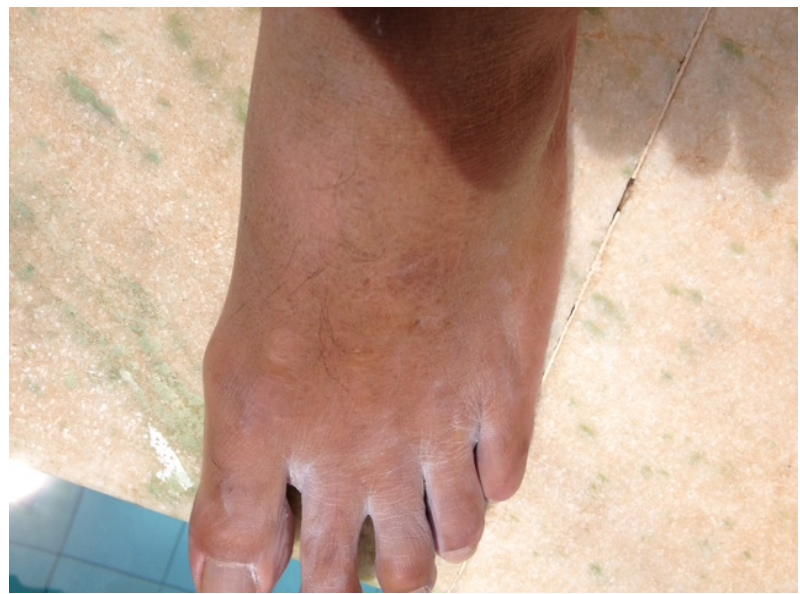

Figure 16. Lesions are noticed between the toes. They were deep with exposed muscles and tendons. No infection, no pain and healing in a short period of time. They were repeated several times. There is presence of drying powder.

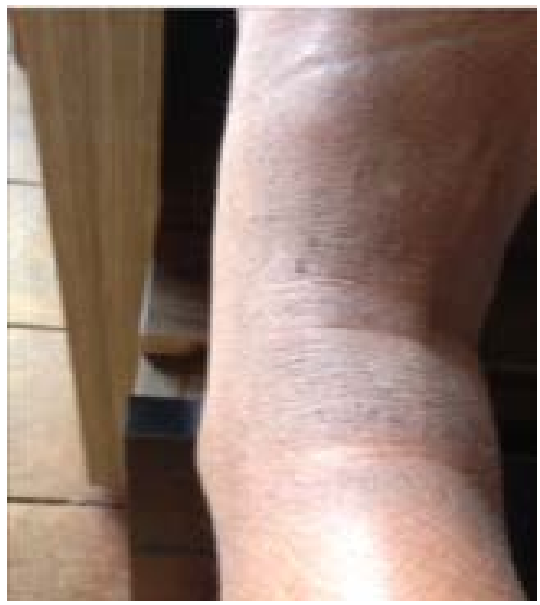

Figure 17. There is a burn with edema in the leg and right foot. 
months, because the same problem arose, if I did! Over the months, it disappeared.

\subsection{General Signs and Symptoms}

Undoubtedly, in general, both the excessive emission of biophotons and the energy deceleration have diverse and harmful effects on all segments of the body. For example, the pain of excessive energy loss is a crushing, tearing pain, while the pain of energy retention is a stabbing pain.

\subsubsection{Pain}

This is the most important and puzzling symptom. Deep, intuitive pain, usually mild in intensity in the calves, for 365 days a year. Here, the biological brain completely ignores pain, so the question is which brain identifies pain and where is it located? Pain is not in matter or flesh itself. It does not give in under the influence of any analgesic drug known to science. Even "morphine" was used at the beginning of the clinical picture. Occasional pain in the bones of the feet and hands, as well as in the trapezius muscle around the back of the neck. Tongue pain, like burning caused by hot milk. There is pain in forearm muscles and pain in the wrist joints.

I once felt the worst pain in my life. For a short time, fortunately. It was caused by excessive loss of biophotons caused by a drug I was still evaluating its effectiveness. This drug is known by science and is very effective in treating deep vein thrombosis. I took 1 pill every 12 and waited for the result. The result was dramatic. Around 2:00 am, I woke up with excruciating pain in my calves.

I already knew that "sleep" was the most important physiological state for balancing the emission of biophotons. It is the most efficient natural "remedy" to relieve this pain. I took midazolam maleate $30 \mathrm{mg}$ orally [double dose] to sleep. It was totally useless. I took $10 \mathrm{mg}$ of "morphine" orally. It was also useless. I reached the top of the stairs in my house and thought. So, all that remains is for me to increase the uptake of biophotons and achieve balance. Like? I looked at the stairs and concluded: maybe going up and down these stairs, several times to relieve the pain. All this happened at dawn. And so it was done. Within $5 \mathrm{mi}-$ nutes the pain disappeared in 50\%; so I continued for I don't know how long. Then I fell into bed and slept for $3 \mathrm{~h}$ maybe. I never repeated the experience again [see treatment topic].

\subsubsection{Bleeding}

Here, hemorrhage is probably due to damage to vascular endothelium due to the action of biophotons. There was no coagulation disorder in the patient, as there was no blood dyscrasia or altered liver function. There were normal laboratory tests. Occasional bleeding in the gums, cheeks and tongue, as well as nose bleeding 365 days a year.

\subsubsection{Muscle and Skin Injuries}

Burns to the skin of the arms and hands, legs and feet, in addition to lesions be- 
tween the fingers, such as cooking with exposure of muscle tendons. Deep lesion in the right calf. The nail falls on the 5th toe of the right foot four times. All this happened without pain, without infection, with good recovery, without sequelae.

\subsubsection{Muscle Fatigue}

This was the worst symptom involving the muscles of the lower limbs [calf] and upper [arm and forearm]. It was as if I ran the Boston Marathon twice daily. It quickly disappeared when the limbs were submerged in a hydro-massage bath or after bathing. There was unchanged lactic acid.

\subsection{Specific Signs and Symptoms}

\subsubsection{Central Nervous System}

Extreme irritability. Change in cognition due to cortical energy saturation, affliction and restlessness during 24 hours.

\subsubsection{Peripheral Nervous System}

Paresis or paresthesia in the palms of the hands and soles of the feet are the main changes in limbs involving the peripheral nervous system, often with falling objects from the hands. Changing the perception of taste as metallic taste, bitter and salty. Alteration of perception in the palms of the hands and soles of the feet with loss of grip and a lot of dryness. Neural pain in the right thigh. Sciatica crisis with continuous "tired" pain due to involvement of the left sciatic nerve. Here, there was an alteration in the electroneuromyography of the lower limbs. Accumulation of biophotons in urine, with urethra spasm and some urinary urgency. The pain was excruciating. Good thing it didn't last more than a minute. It is noteworthy that all these changes must be due to involvement of peripheral nerves or increased concentration of biophotons in urine.

\subsection{Laboratory Test Results}

\subsubsection{Fasting Blood Glucose}

I do daily physical activity. Walk. I am $1.70 \mathrm{~m}$ tall and weigh $78 \mathrm{~kg}$. Fasting blood glucose was always normal. In 2014, when the first symptoms of this syndrome appeared, fasting blood glucose was $88 \mathrm{mg} / \mathrm{dl}$. In 2015, it was already 107 $\mathrm{mg} / \mathrm{dl}$. In 2018 it rose to $111 \mathrm{mg} / \mathrm{dl}$. The glycated hemoglobin in 2018 was 5.7\%. The previous dosage was $5.2 \%$. In 2019, it increased to $127 \mathrm{mg} / \mathrm{dL}$, then reduced to $113 \mathrm{mg} / \mathrm{dL}$ and the last dosage was $117 \mathrm{mg} / \mathrm{dL}$. Following the chart, there is a dangerous rise in blood glucose, despite some fluctuations. At the moment, I'm just glucose intolerant (see Graphic 1).

\subsubsection{B12 Vitamin}

In 2014, when the clinical picture appeared, several months later, vitamin B12 was low, at a critical level with $152 \mathrm{pg} / \mathrm{mL}$ and already showing some symptoms. Treatment, divided into a 7-day series, for 3 months was suggested. Then, around 4 months, another dosage was performed; whose value was $522 \mathrm{pg} / \mathrm{mL}$. There was no subsequent treatment. In 2015, there was a downward oscillation, 


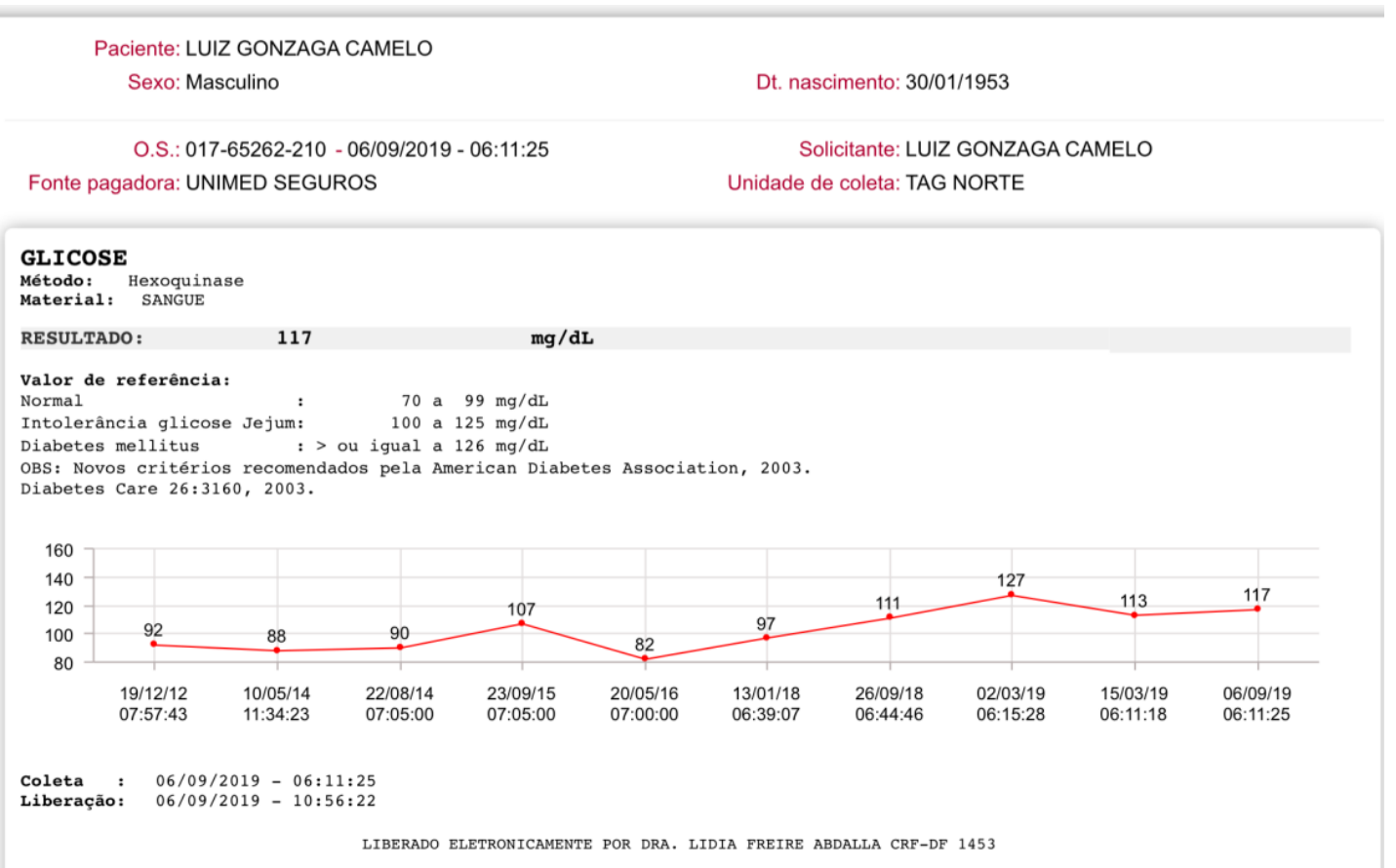

Graphic 1. Oscillatory evolution of fasting glucose for more or less from 2012, two years before the onset of the clinical picture.

with a value of $210 \mathrm{pg} / \mathrm{mL}$, still within the normal range and without prior treatment. There was an increase in subsequent years until reaching $664 \mathrm{pg} / \mathrm{ml}$ in 2018 with occasional treatment. In 2019, there was a reduction to $251 \mathrm{pg} / \mathrm{ml}$, still within the normal range. No treatment (see Graphic 2).

\subsubsection{Lipidogram}

\section{1) Total cholesterol}

In 2014, the cholesterol dosage was $141 \mathrm{mg} / \mathrm{dL}$. In 2015, the dosage was 187 $\mathrm{mg} / \mathrm{dL}$. In 2016, it had a critical rise to $241 \mathrm{mg} / \mathrm{dl}$, with fluctuations more or less in the following years until it remained at $227 \mathrm{mg} / \mathrm{dL}$, already in 2019 considered a very high value. Up to this time without treatment (see Graphic 3).

\section{2) Triglycerides}

In 2014, the triglyceride dosage was $128 \mathrm{mg} / \mathrm{dL}$, while in 2015 it rose to 226 $\mathrm{mg} / \mathrm{dL}$; then $278 \mathrm{mg} / \mathrm{dL}$, in 2016, with fluctuations up or down until it remained at $281 \mathrm{mg} / \mathrm{dL}$ in 2019 , a very critical increase. Up to this time without treatment (see Graphic 3).

\section{3) HDL cholesterol}

In 2014, the dosage was $33 \mathrm{mg} / \mathrm{dL}$, with little variation for more or less until it remained at $51 \mathrm{mg} / \mathrm{dL}$, in the year 2019 . There is a healthy increase. At that time, I was walking daily with the aim of eliminating excess energy. The relief was remarkable, however, after 2 hours, the energy returned as before (see Graphic 3).

\section{4) LDL cholesterol [bad cholesterol]}

In 2014 , the dosage was $82 \mathrm{mg} / \mathrm{dL}$, until it remained dangerously at $135 \mathrm{mg} / \mathrm{dL}$ in 2019 (see Graphic 3). 
VITAMINA B12

Método: Quimioluminescência Material: SORO
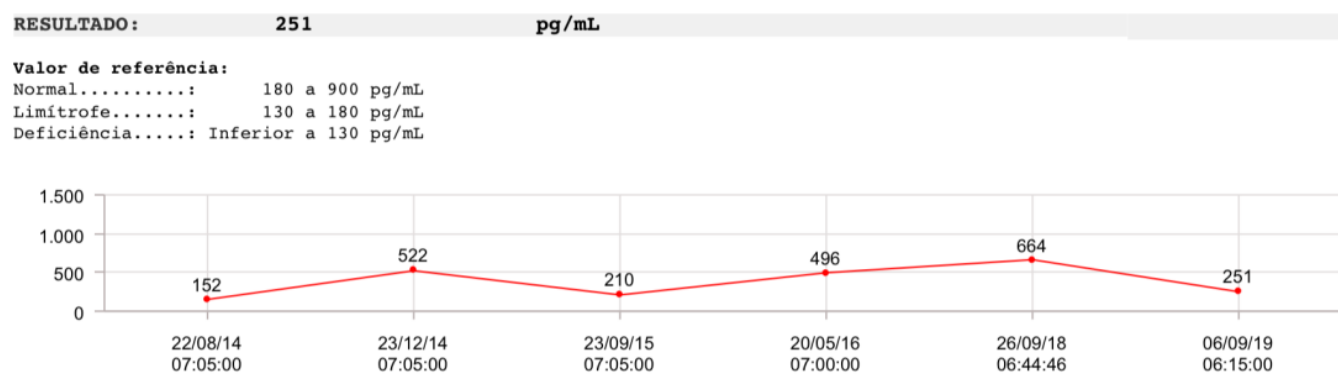

Coleta : $\quad 06 / 09 / 2019-06: 15: 00$
Liberação:

LIBERADO ELETRONICAMENTE POR DRA. LIDIA FREIRE ABDALLA CRF-DF 1453

Graphic 2. Oscillatory evolution of vitamin B12 dosage from 2014, at the beginning of the clinical picture, until 2019.

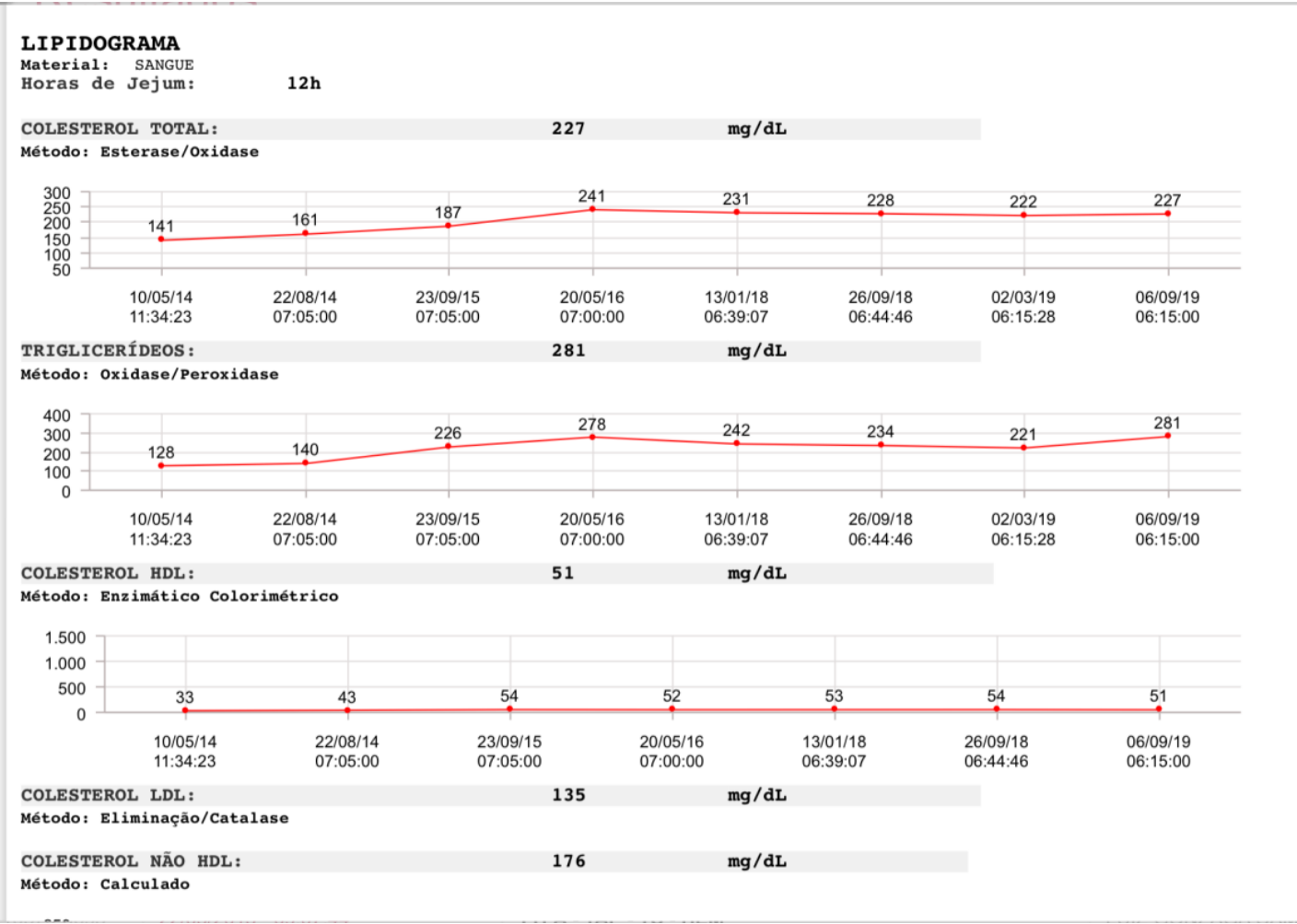

Graphic 3. Oscillatory evolution of Lipidogram dosage from 2014 to 2019.

\subsubsection{Total Testosterone}

In 2015, with a year of evolution of the condition, total testosterone presented a dosage of $343.6 \mathrm{ng} / \mathrm{dL}$ considered normal. In 3 years it rose to $413.54 \mathrm{ng} / \mathrm{dL}$, an increase of $17 \%$, without any prior treatment. In 2019, it increased to 426.21 $\mathrm{ng} / \mathrm{dL}$, characterizing a significant increase over 4 years (see Graphic 4).

In Table 1, here, there could be numerous characteristics; however, those that most caused transient morbidity were mentioned.

In Table 2, most common features are noted. 
TESTOSTERONA TOTAL

Material: SORO

RESUlTADO:

426,21

valor de referência

Adul tos:

Masculino: $21-49$ anos: 164,94 a $753,38 \mathrm{ng} / \mathrm{dr}$

50 - 89 anos: 86,49 a $788,22 \mathrm{ng} / \mathrm{dI}$

Feminino: Pré-menopausa

21 - 60 anos: 12,09 a $59,46 \mathrm{ng} / \mathrm{dL}$

Pós-menopausa

Pediátricos:

Masculino: 2 a 10 anos: $<7,00$ a $25,91 \mathrm{ng} / \mathrm{dL}$

11 anos: $:>7,00$ a $341,53 \mathrm{ng} / \mathrm{dL}$

12 anos $\quad: \quad<7,00$ a $562,59 \mathrm{ng} / \mathrm{dL}$

14 anos : 23,28 a $742,46 \mathrm{ng} / \mathrm{dL}$

15 anos : 144,15 a $841,44 \mathrm{ng} / \mathrm{dL}$

16 a 20 anos: 118,22 a $948,56 \mathrm{ng} / \mathrm{dI}$

Feminino: 2 a 10 anos: $<7,00$ a $108,30 \mathrm{ng} / \mathrm{dL}$

11 a 15 anos: $<7,00$ a $48,40 \mathrm{ng} / \mathrm{dL}$

16 a 20 anos: 17,55 a $50,41 \mathrm{ng} / \mathrm{dL}$

*Atenção aos novos valores de referência a partir de 04/08/2018 - Análise Equipamento Advia CentaurXP» - Testosterona II

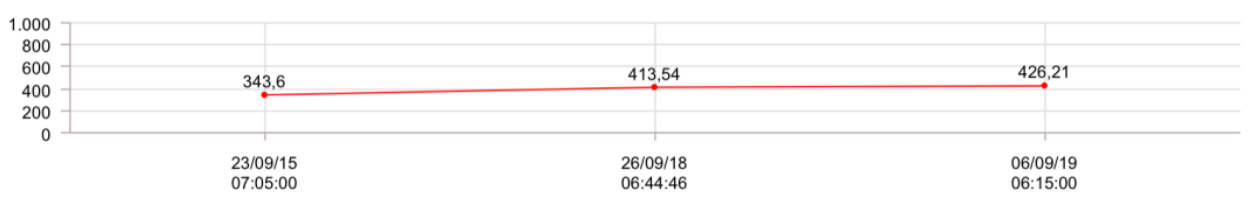

Graphic 4. Evolution of total testosterone dosage from 2015 to 2019.

Table 1. Most relevant clinical characteristics.

Domain: Patient characteristics

Sciatica pain in left leg

Irritability

Biophoton emission from the eyes

Nasal obstruction

Pseudoparalysis in cheeks with biting and bleeding

Tongue burn

Bleeding of gums and nasal mucosa

Increased serum glucose

Increased triglycerides and cholesterol

Testosterone increase

Right saphenous vein lesion with surgical removal

Excessive biophoton emission at the beginning of the clinical picture

Table 2. Most relevant sociodemographic characteristics.

Domain: Patient characteristics

Male, 60 years old

Education level: higher

Marital status: married

Family arrangement: resides with someone

Paid work: retired

Monthly Income: \$10,000

Residence: urban area

Health status: no comorbidity 


\subsection{Procedures Performed}

Follows the chronological evolution of the procedures performed over the time of patient follow-up.

\subsubsection{Description of Limb Electroneuromyography}

This test was performed over three years of patient follow-up, from 2014 to 2017.

1) First test: five months after the onset of the clinical condition, on 05.22.2014

- The electromyogram did not reveal changes in the assessed muscles;

- Sensory responses of the superficial peroneal nerve with reduced amplitudes and prolonged latencies. Inexcitable sural nerve sensory responses;

- Reduced or absent sensitive conduction speeds in lower limbs.

Conclusion: These findings demonstrated axonal and demyelinating sensory denervation only in the lower limbs, compatible with moderate peripheral polyneuropathy. Upper limbs are unchanged.

2) Second test: three years after the onset of the clinical condition, on 06.10.2017

- Electromyography of the four limbs within normal limits. Spontaneous resolution.

\subsubsection{Description of Color Venous Echodoppler of Lower Limbs}

The color venous Doppler test, consisting of three tests in all, was performed during patient follow-up from 2015 to 2017.

1) First test: one year after the beginning of the clinical condition, on 02.19.2015.

Conclusion:

Right lower limb.

- Pervious deep venous system, competent and without signs of deep vein thrombosis.

- Safena magna patent, with segmental reflux.

- Competent and non-refluxing small perveal safena.

- Medium-caliber varicose veins with reflux in the right thigh and leg Left lower limb.

- Pervious deep venous system, competent and without reflux, without signs of deep vein thrombosis.

2) Second test: seven months after the first test, on 09.15.2015: right and left lower limb.

Conclusion: Lower right limb and lower left limb.

- Pervious venous system, competent and without reflux, without signs of deep vein thrombosis.

- Safena magna pervea, with full reflux in the right leg.

- Perveal parva safena, competent and without reflux in the right lower limb.

- Medium-caliber varicose veins with reflux in the right thigh and leg. 
- Medium-caliber varicose veins with reflux in the left thigh and leg [here, the first test was unchanged].

3) Third test: two years after the second exam, on 09.16.2017: right and left lower limb.

Conclusion: Lower right limb and lower left limb.

- Pervious deep venous system, competent and without reflux, without signs of deep vein thrombosis.

- Safena magna pervea, with full reflux.

- Medium-caliber varicose veins with reflux in the right lower limb.

- Medium-caliber varicose veins with reflux in the left lower limb.

\subsection{Description of Lower Limb Arterial Color Echodoppler}

This test was always unchanged throughout the patient's follow-up.

Technique used: Duplex Scan [Echography and Doppler] of the Arterial Vascular System of the lower limbs. Used GE Logiq P5 device.

Conclusion: Lower right and lower left limb [02.19.2015 and 09.15.2015].

- Pervious arterial system, with normal flow and no stenosis or obstructions.

Attention: After two years of evolution there was no change in the right Safena Magna Vein. The injury that appeared in the second test remained in the third. So an invasive procedure via Endolaser was indicated.

Review: Description of color venous echodoppler of lower limbs: here, I made the description putting the items $(1,2$ and 3$)$ in sequence.

\section{Discussion}

The emission of biophotons follows a pattern that varies in intensity, in all segments of the body. In fact, in this study by sequence, the highest emissions occurred in the hands and feet, head region, upper $1 / 3$ of the chest and neck, and finally in the genitalia.

The results of the current study showed that the intensity of biophoton emission varied significantly during the day. It was variable during the morning, afternoon and evening, characterizing a biological rhythm, that is, a standard physiological rhythm. From five o'clock in the morning, it started to increase with the beginning of daily activities and throughout the morning; it increased more in the afternoon and even more in the evening until completing 24 hours around 5:00 the next day. The origin of such fluctuations is unknown. Presumably, it must be a common characteristic pattern of biophoton emission in humans [2] including, without a doubt, a strong influence of the geomagnetic field, the seasonal rhythm and perhaps the circadian rhythm [18].

Previous studies have also shown variation in the intensity of biophoton emission. As demonstrated, it started with weak emission in the early morning [2], increased in intensity throughout the morning, with a peak in the afternoon [2] and even higher in the early evening and remained high during the night [29] [32] [33]. It is noteworthy that the correlation with geomagnetic activity [7] [14] 
[33] [34] [35], that is, planetary magnetic influence, was remarkable, however, there are studies that refer to the circadian rhythm [32], metabolic processes and regulation of biological activity [7]. However, the circadian rhythm follows a well-known pattern. It starts around 3:00 am, increases during the morning and begins to reduce after noon; continues decreasing until early evening, until it stabilizes at baseline.

It is important to emphasize that, according to some studies, there is no uniformity in the distribution of biophotons throughout the body. The current study exhibited a common pattern, emphasizing the extremities [region of the head, hands, feet, and genitalia] as sites of greater biophoton emission, presumably due to the greater density of blood vessels, characteristically in these anatomical regions. Undoubtedly, there is a common pattern of biophoton emission in humans [2] [36]. The hands and head region emit the highest levels of biophotons [2] [18] [21] [30] [36]. In our study, in addition to burning the right auricule, the emission of biophotons was significant in hair, forehead, ears and especially eyes, mouth and nose. In the nose, in addition to the intense nasal obstruction to date, there was hemorrhage for several years and loss of nasal mucosa tissue. In the eyes, the biophoton emission is the most intense and "enigmatic" in the human body, perhaps because they are very sensitive organs and because it is a pathological emission. In fact, the highest levels occurred in the forehead and hands region, and lowest in the abdomen and back [2] [23]; higher around the mouth and cheeks, in fingertips [36] and right hand and also higher during the night [30] which is in agreement with other studies [18] [22] [25] and almost double the emission in the palmar region also in agreement with the current literature [18] [34] [37].

It is important to highlight that higher emissions were observed around the mouth, cheeks and eye sockets [2] [20] [22] [25] [30] [38], head and hands in the palmar and dorsal region [18] [30] [38]. Undoubtedly, in the current study, biophoton emission was so severe in the mouth that it led to pseudoparalysis in the right cheek and frequent bite with tissue loss, as well as bite in the right lip, in addition to gingival hemorrhage and tongue burn. Here I believe there is peripheral nerve involvement leading to cheek pseudoparalysis.

In the current study, there is a remarkable asymmetry in the emission of biophotons, predominantly in the entire right side of the body. In fact, the emission on the right side is higher than on the left side [30] [37]. Certainly, it is speculated that this fact is attributed to individual differences or because they are right-handed individuals [32]. As demonstrated in another study, the increase in intracerebral photon emission is correlated with the planetary magnetic field from the left hemisphere and not from the right [33]. It is worth remembering that, considering the inversion of cerebral hemispheric axes and that, therefore, the left production is emitted through the right side, this may be the reason for the high emission of biophotons from the right side of the patient's body [33]. Left-right asymmetry fluctuated daily; in the right hand, the emission was higher 
than in the left during the day [30] [32], while at night it was lower [30] [37], a fact that is in disagreement with the current study that assures it is quite high at night, corroborated by another study [36]. On the other hand, other studies reported symmetry of the left-right hand and not in the dorsal-ventral region of the human body, including elbows, hands and knees [20] [35] [36]. However, in later experiments with more individuals, this difference in left-right symmetry was not proven [22] or is relatively small [30]. However, other studies have shown right-left and dorsal-ventral symmetry [36] [37]. It is also a fact that this symmetry changes during the night, perhaps due to a regulatory mechanism in the metabolism of biophotons [30] [32].

As described above in the current study, the distribution and intensity of biophoton emission throughout the body were not uniform. The emission was higher in the palms of the hands, especially the right hand, fingertips, with greater intensity in the middle, index and thumb fingers (see Figures 3-5). These data were confirmed in other studies [2] [18] [22] [30] [32], however, in the dorsal region of the fingers, there was a reduction in the emission of biophotons [22], which is in agreement with what we believe. In fact, we believe it to be due to few blood vessels in the dorsal region of the hand and high vascular density in the palmar region and, in general, in other extremities of the human body. As a result, greater emission of biophotons from the fingertips is characterized. Here, it is worth noting that the hematogenous route is the main transport vehicle for biophotons.

On the other hand, our study did not show a significant increase in biophoton emission on the back of the hands, unlike other studies [30] [32]. This discrepancy may be due to thicker blood vessels on the back of the hands (see Figure 9), which could characterize a false positive measurement result found in other studies [30] [32], given the high sensitivity of electromagnetic devices. It should also be noted that the toenails and fingernails produce higher emissions [2] [22] [32] and also the fingertips [2] [30] [32] [36], since there was detachment of already devitalized tissue under the nails, especially the foot right, in which severe injury with loss of a nail occurred, demonstrated in the current study (see Figure 4, Figure 11 and Figure 13). Some studies suggest that sick patients emit more biophotons [22]. However, the patient who writes to you with the highest emission of biophotons, considered pathological and not registered in the medical literature, has never been as healthy as he is now, except for the alterations observed in this study.

Surprisingly, the current study has shown rejuvenation and increased serum testosterone levels and a considerable increase in libido. Of course, that's not the rule. In fact, in the sick patient, there is a break in symmetry and quantum coherence of the biological organizing model, while the disease persists.

It is noteworthy that previous studies have shown a strong restriction of blood flow with the use of tourniquets in the hands and the consequent reduction in the emission of biophotons [38], as well as a reduction in the temperature of the ischemic area [39]. Therefore, I believe that this fact is due to the reduction of 
cellular metabolism in the ischemic region. Undoubtedly, this supposed mechanism was indirectly observed in the current study, when there was a significant reduction in the emission of biophotons after the submersion of hands in a basin with ice water, with considerable improvement for some time, denoting blood flow restriction. Here, it seems to suggest that blood has some peculiar mechanism that detects temporal variations in temperature [39] [40]. The reasons for this event are unknown.

It is observed in this study that the emission of biophotons follows an invariable chronology that is correlated with climatic and seasonal variations. As highlighted above, several studies have shown the existence of a physiological and seasonal rhythm in the emission of biophotons common to every human being [18] [41]. In the current study, there was a significant reduction in the emission of biophotons in the winter and spring months and a very strong increase in the summer and autumn months. This chronology is also in agreement with previous study data [34] [35] [36] [42], whereas other studies have shown a significant reduction in the autumn months [18] [20] [38] [43]. The reasons for these events are unknown.

There seems to be an influence of external factors in contact with the skin on biophoton emission. At the onset of symptoms, when biophoton emission was excessive, there was a reduction in that with significant relief when the hands were submerged in a basin of ice water. This fact is certainly due to the reduction in blood flow through the ice and the consequent reduction in the emission of biophotons due to vasoconstriction and alteration of cell metabolism in the ischemic region [39]. In fact, these data were also demonstrated by Nakamura and Hiramatsu in a previous study [44].

Since there is quantum coherence in human brains predicted by quantum field theory [1] [4] [15] [28] it makes sense to emphasize that photons are kept coherently throughout the organism [9] [12] [20] [45]. In fact, with the sudden exposure to sunlight and the consequent absorption of photons described in the current study, there are strong correlations with high biophoton emission several orders of magnitude above what would be expected for their spontaneous emission, which was corroborated in another study [45]. Undoubtedly, as reported above, it is unequivocal proof of the absorption and conversion of photons into biophotons, instantly, by living beings, including man.

The most relevant metabolic changes were in glucose, vitamin B12, triglycerides, cholesterol and testosterone. Fasting glucose ranged from $111-129 \mathrm{mg} / \mathrm{dl}$, characterizing glucose intolerance. Therefore, I decided to take preventive treatment with "sitagliptin phosphate" $50 \mathrm{mg}$ per day, orally. Here, the treatment is done every other day, in order not to worsen the symptoms due to excessive loss. It is noteworthy that possibly biophotons, glucose and cholesterol are closely correlated to the point of sharing the same metabolic pathway and biochemical processes. Thus, it is assumed that there is synchronicity in the metabolism of biophotons, glucose and cholesterol. 
With the introduction of hyperglycemia treatment, circulating glucose returns to the cells, increases intracellular activity by increasing the supply of glucose into the cells and thus supposedly increasing the synthesis of biophotons. With the increase in the concentration of biophotons, there is retention and, finally, the appearance of symptoms. Here, these symptoms are noticed in the first two hours of the drug's action, with the appearance of facial vasodilatation, redness and localized temperature increase, nasal obstruction and greater excretion of biophotons through the eyes. In this context, these symptoms only disappear when there is an increase in the excretion of biophotons through normal physiological pathways after 2 hours of drug action. However, in the remaining twelve hours, due to the retention of biophotons, resulting from the excessive loss due to the treatment of hyperglycemia, the general symptoms worsen. On the other hand, something similar also occurs with the treatment of hypercholesterolemia. See more details in the part 2 edition of this essay still in preparation describing the evolution of this syndrome. It is noteworthy however that glucose, cholesterol and biophotons are forms of "vital energy" supposedly arising from the metabolism of photons.

Finally, it is important to remember that there was a delay in aging and a significant increase in libido. In fact, both are certainly due to the fact that biophoton is a "vital energy", in addition to the significant increase in serum testosterone levels.

\section{Treatment}

All treatment is palliative. There is no specific treatment, however, over these seven years I accumulated experience and I made some discoveries.

\subsection{Hydrotherapy}

At the beginning of the excessive emission phase of biophotons, due to lack of knowledge, I was very afraid of losing the ends of my feet, due to an eventual burn or cooking. So I put them in a basin of water to avoid supposed injuries and also because it brought great relief. All of this was mostly done at night. The feet did not show any abnormalities from this procedure. In this way, I could also observe, throughout the night, the geomagnetic action in the emission of biophotons, whose worsening of symptoms extended until around 5:00 am.

Water, a universal solvent, is also the most efficient conductor of electricity. Soon, I identified that I was facing the fastest and lowest cost therapy practice against the harmful action of the pathological emission of biophotons. Unfortunately, the positive effect is short-lived. It is the most efficient therapy in all phases of biophoton emission, but it can also cause excessive energy loss. There needs to be balanced, both during the "bath" and in the submersion of body segments. Then came the idea of submerging the hands up to the middle third of the forearm in a basin of water. The improvement was instantaneous, and even better with cold water, very cold. Sometimes I would add ice and keep my hands 
in a basin of water for 2 - 3 minutes. There is a total absence of cyanosis. Vasodilatation by the action of biophotons was so great that, when I took my hands out of the basin, they were red, well colored. The perception was that it eliminated energy from the entire body, especially the hands and feet. After an hour, everything came back!

\subsection{Diosmin [Diosmin $450 \mathrm{mg}+$ Hesperidin $50 \mathrm{mg}$ ]}

This drug acts on the veins of the circulatory system, decreasing venous stasis, improving venous tone, increases the dynamic force of blood within vessels. It is used to treat deep vein thrombosis. Physiologically, by perception, it leads to an agitation of the blood inside the vessels. Therefore, it increases the "excretion" of biophotons mainly in the energy retention or accumulation phase. It is excellent at a dose of $500 \mathrm{mg}$ once a day and at all stages of biophoton emission. The positive effect starts quickly, somewhere around 2 to 4 hours and lasts around 20 hours. Like other types of treatments, there is also the possibility of excessive energy loss. The only time I tested a pill twice a day I had the 'worst' pain in my life from excessive loss of biophotons. I never repeated the operation again. See the pain topic.

\subsection{Moringa Oleifera}

Considered nature's green meat, moringa oleifera contains 47 antioxidants. They were extracted in extracts from leaves, bark, roots and flowers in the laboratory and are considered one of the best natural antioxidants widely used in traditional medicine [46]. Used by me without any pretense, for other purposes, I found beneficial effect on the excretion of biophotons every time I took a $500 \mathrm{mg}$ capsule a day. It was tested at the end of the process, in the energy retention phase, when it is more difficult to eliminate it naturally. It presents a very physiological excretion of biophotons during the twenty-four hours of the day, relieving symptoms. As in other types of treatment, there is a certain energy loss with the appearance of some disorders, without much significance. It has great potential for use in the excessive emission phase of biophotons. I didn't use more than one capsule a day for fear of possible increase in pain or appearance of other symptoms. There needs to be a balance.

\subsection{Naphazoline Hydrochloride $0.5 \mathrm{mg} / \mathrm{ml}$ [In Equal Parts with $0.9 \%$ Sodium Chloride Solution]}

It is noteworthy that the action of biophoton causing vasodilatation is unprecedented in medicine. When excreted through the nostrils, it causes dilation in the nasal microvessels with intense dryness of the mucosa. Naphazoline is a powerful vasoconstrictor used in nasal obstruction. Due to its prolonged use, I was prudent in diluting it with "saline solution" so that drug-induced rhinitis would not occur. It was used several times a day, mostly at night. Improvement was almost always rapid however when vasodilatation was of great intensity, it had 
no positive effect.

\subsection{Water Spray on Eyelids and Eyelashes}

Water can mainly be used by "spraying" it on the eyelids and eyelashes. Of course, you should close your eyes to avoid pain and irritation. Use several times a day for 24 hours. Improvement is instantaneous. It is the most effective therapy. It can also be used by washing the eyes.

\subsection{Magnesium Hydrochloride}

Magnesium is one of the body's electrolytes and minerals that carry an electrical charge when dissolved in body fluids such as blood. It is the fourth most abundant mineral in the body. It is required for all enzymatic processes involving ATP and also for many enzymes involved in nucleic acid metabolism. Several enzymes are activated or dependent on magnesium. It can be used in vitamin and mineral supplements or alone. Here, it was used to increase the excretion of biophotons, mainly in the energy retention phase. It possibly prevents muscle myofibril contractions and consequent pain.

\subsection{Tramadol Hydrochloride}

It is a powerful analgesic used up to every 6 hours for moderate to severe pain, especially after trauma and very invasive surgeries. In the current case, it was not necessarily used as an analgesic. Here it was used to reduce the excretion of biophotons, particularly in the excessive emission phase, as a $50 \mathrm{mg}$ capsule once a day. Can be used more than once, but causes excessive energy retention with worsening of some symptoms.

\section{Conclusions and Perspectives}

It should be noted that the "mind" is a fluidic body of high vibratory frequency that serves as a vehicle for the manifestation of thought. Here, it is clear that the agent that plays the most relevant role in the emission of biophotons is the Psychosome. While science does not chase in search of that "powerful agent," multi-dimensional we still would go for a good time in the pre-Enlightenment darkness. It's a prediction, a prognosis. It's hard to believe that the biological brain, a greasy, friable and bad tasting organ, is capable of generating "consciousness", something so extraordinary and inconceivable. Until science learns to deal with the Psychosome, knowledge about the emission of biophotons will remain at the same level as they are. The emission of biophotons follows an intelligent and intentional pattern, all the time.

Over the course of this phenomenon, the emission was of high intensity across the right side of the body, leaving behind a trail of destruction in the form of extremely relevant injuries. Suddenly, at the end of the process, it reversed this tendency and began to emit more intensely on the left side of the body, with notable lesions such as skin burns, increased emission of biophotons from the 
left eye and deep fissures in the heels. It seemed the right side communicated with the left and knew what he was doing. The pain, for example, was always stronger in the left calf muscle, while the saphenous vein lesion was in the right calf muscle. Another very relevant situation was the "slowdown" of the emission of biophotons, whose duration was over three years. In fact, she was cautious and methodical throughout. If not I would have died.

Throughout this energetic disturbance, energy imbalance and/or rebalancing were observed on several occasions, both due to the use of drugs under test and the therapeutic use of water. For any deviation, my body entered the process of "energy self-defense". Here, what matters is balance. Given this information, it is noteworthy that, without a doubt, biophoton should be considered an effective "biomarker" that can also be used to measure the existence of balance/imbalance in energy distribution or biophoton. A very convincing geomagnetic rhythm was described at various periods during the twenty-four hours, including worsening of symptoms, especially in the afternoon and evening.

Regarding the treatment, at the end of the process, there was almost always an excessive loss of biophotons and the consequent negative reaction of the body, both to hydrotherapy and to the other drugs tested, causing mild discomfort that stabilized within 24 hours. In hydrotherapy, however, energy rebalancing occurred within a few minutes.

In closing, I take the liberty of using some relativistic concepts to explain what happened to me. Matter is limited and very slow to explain everything. When the intelligent being enters the scene, it gains the speed of light and changes everything. However, the cell itself is not intelligent, but is radiated by the "relative intelligence" of its holder, which, in turn, triggers its intrinsic mechanisms. For example, who absorbs oxygen? It is the intelligent being of the universe, man, who is the holder of cellular life. So what is life? It is consciousness covered by matter. It is she, therefore, who triggers the mechanisms necessary to carry out "biological functions" and who, in fact, consumes oxygen. Who makes oxygen available? The Supreme Intelligence of the Universe, the primary cause of all things.

So, to be coherent, it happens with the photon. It is perennial in nature. Because it is intelligent, it is converted into biophotons and thus absorbed, fulfilling its main role in the body. And how are other living beings in this scenario? It has rudimentary intelligence, an intelligent principle, on the way to individualization, linked to Universal Consciousness, which is in accordance with quantum field mechanics. Everything in the universe has the same fluidic origin, except for the Intelligent Being. In fact, what really changes is the vibratory frequency of the "primitive or primordial elemental matter" that is found somewhere, possibly in the quantum vacuum.

In fact, everything in the universe is intelligent and coherent [3]. What seems random to us is ultimately an intelligent effect. For example, let's think about what might happen inside an atom. So only intelligent effect! A "hamster" cell is intelligent [3]. It performs thousands of chemical reactions a day and cannot go 
wrong. The plant cell, on the other hand, responsible for the metabolism of the "Moringa oleifera" [46] is intelligent [3] and generous, as it regulates its own energy supply, keeps the [toxic] $\mathrm{CO}_{2}$ and releases the [healthy] $\mathrm{O}_{2}$ for others. By discovering the diagnostic and especially healing properties of biophoton, we will enter a new scientific level where we will deal with a new investigative and therapeutic science. I assume it is necessary to create electromagnetic optical depth vision devices. It will be the beginning of subtle therapy on a grand scale.

\section{Conflicts of Interest}

The author declares no conflicts of interest regarding the publication of this paper.

\section{References}

[1] Rahnama, M., et al. (2011) Emission of Mitochondrial Biophotons and Their Effect on Electrical Activity of Membrane via Microtubules. Journal of Integrative Neuroscience, 10, 65-88. https://doi.org/10.1142/S0219635211002622

[2] Van Wijk, E.P.A., et al. (2006) Anatomic Characterization of Human Ultra-Weak Photon Emission in Practitioners of Transcendental Meditation ${ }^{\mathrm{Tm}}$ and Control Subjects. The Journal of Alternative and Complementary Medicine, 12, 31-38. https://doi.org/10.1089/acm.2006.12.31

[3] Camelo, L.G. (2021) Existential Conflicts of Neuroscience: A Brief Analysis of Consciousness. Journal of Consciousness Exploration \& Research, 12, 23-31.

[4] Salari, V., et al. (2015) Ultraweak Photon Emission in the Brain. Journal of Integrative Neuroscience, 14, 419-429. https://doi.org/10.1142/S0219635215300012

[5] Bischof, M. (2000) Field Concepts and the Emergence of a Holistic Biophysics. In: Beloussov, L.V., Popp, F.A., Voeikov, V.L. and Van Wijk, R., Eds., Biophotonics and Coherent Systems, Moscow University Press, Moscow, 1-25.

[6] Curtis, B.D. and Hurtak, J.J. (2004) Consciousness and Quantum Information Processing: Uncovering the Foundation for a Medicine of Light. The Journal of Alternative and Complementary Medicine, 10, 27-39. https://doi.org/10.1089/107555304322848931

[7] Rubik, B. (2002) The Biofield Hypothesis: Its Biophysical Basis and Role in Medicine. The Journal of Alternative and Complementary Medicine, 8, 703-717. https://doi.org/10.1089/10755530260511711

[8] Meijer, D.K.F. and Geesink, H.J.H. (2017) Consciousness in the Universe Is Scale Invariant and Implies an Event Horizon of the Human Brain. NeuroQuantology, 15, 41-79. https://doi.org/10.14704/nq.2017.15.3.1079

[9] Bischof, M. (2008) Synchronization and Coherence as an Organizing Principle in the Organism, Social Interaction, and Consciousness. NeuroQuantology, 6, 440-451. https://doi.org/10.14704/nq.2008.6.4.200

[10] Rubik, B., et al. (2015) Biofield Science and Healing: History, Terminology, and Concepts. Global Advances in Health and Medicine, 4, 8-14. https://doi.org/10.7453/gahmj.2015.038.suppl

[11] Kofatos, M.C. (2015) Biofield Science: Current Physics Perspectives. Global Advances in Health and Medicine, 4, 25-34.

https://doi.org/10.7453/gahmj.2015.011.suppl 
[12] Bischof, M. (2003) Introduction to Integrative Biophysics. In: Popp, F.A. and Beloussov, L., Eds., Integrative Biophysics, Springer, Dordrecht, 1-115. https://doi.org/10.1007/978-94-017-0373-4_1

[13] Bohm, D. (1990) A New Theory of the Relationship of Mind and Matter. Philosophical Psychology, 3, 271-286. https://doi.org/10.1080/09515089008573004

[14] Rubik, B. (1995) Energy Medicine and the Unifying Concept of Information. Alternative Therapies in Health and Medicine, 1, 34-39.

[15] Bhaumik, M. (2014) Is the Source of Awareness Present in the Quantum Vacuum? In: Menon, S., Sinha, A. and Sreekantan, B., Eds., Interdisciplinary Perspectives on Consciousness and the Self, Springer, New Delhi, 243-252. https://doi.org/10.1007/978-81-322-1587-5_18

[16] $\mathrm{Hu}, \mathrm{H}$. and $\mathrm{Wu}, \mathrm{M}$. (2013) The Relationship between Human Consciousness \& Universal Consciousness. Scientific GOD Journal, 4, 209-225.

[17] $\mathrm{Hu}, \mathrm{H}$. and $\mathrm{Wu}, \mathrm{M}$. (2013) Human Consciousness as Limited Version of Universal Consciousness. Journal of Consciousness Exploration \& Research, 4, 52-68.

[18] Rubik, B. and Jabs, H. (2017) Effects of Intention, Energy Healing, and Mind-Body States of Biophoton Emission. Cosmos and History: The Journal of Natural and Social Philosophy, 13, 227-247.

[19] Bradley, R.T. (2007) The Psychophysiology of Intuition: A Quantum-Holographic Theory of Nonlocal Communication. World Futures, 63, 61-97. https://doi.org/10.1080/02604020601123148

[20] Salari, V., Rahnama, M. and Tuszynski, J.A. (2012) Dissipationless Transfer of Visual Information from Retina of the Primary Visual Cortex in the Human Brain. Activitas Nervosa Superior, 54, 26-48. https://doi.org/10.1007/BF03379582

[21] Meijer, D.K.F. (2012) The Information Universe: On the Missing Link in Concepts of Architecture of Reality. Syntropy Journal, 1, 1-64.

[22] Van Wijk, R., Soh, K.S. and Van Wijk, E.P.A. (2007) Anatomic Characterization of Acupuncture System and Ultra-Weak Photon Emission. Asian Journal of Physics, 16, 443-474.

[23] Soh, K.S. (2004) Bonghan Duct and Acupuncture Meridian as Optical Channel of Biophoton. Journal of the Korean Physical Society, 45, 1196-1198.

[24] Cacha, L.A. and Poznanski, R.R. (2014) Genomic Instantiation of Consciousness in Neurons through a Biophoton Field Theory. Journal of Integrative Neuroscience, 13, 253-292. https://doi.org/10.1142/S0219635214400081

[25] Sidorov, L. and Chen, K. (2012) Biophysical Mechanisms of Genetic Regulation: Is There a Link to Mind-Body Healing? DNA Decipher Journal, 2, 177-205.

[26] Moga, M.M. and Bengston, W.F. (2010) Anomalous Magnetic Field Activity during a Bioenergy Healing Experiment. Journal of Scientific Exploration, 3, 397-410.

[27] Gariaev, P.P., et al. (2011) DNA as Basis for Quantum Biocumputer. DNA Decipher Journal, 1, 25-46.

[28] Bókkon, I., Salari, V., Tuszynski, J.A. and Antal, I. (2010) Estimation of the Number of Biophotons Involved in the Visual Perception of a Single-Object Image: Biophotons Intensity Can Be Considerably Higher Inside Cell than Outside. Journal of Photochemistry and Photobiology B: Biology, 100, 160-166.

https://doi.org/10.1016/j.jphotobiol.2010.06.001

[29] Yang, M.N., et al. (2019) A Bridge of Light: Toward Chinese and Western Medicine Perspectives through Ultraweak Photon Emissions. Global Advances in Health and 
Medicine, 8, 1-7. https://doi.org/10.1177/2164956119855930

[30] Van Wijk, E.P.A., Van Wijk, R. and Cifra, M. (2007) Spontaneous Ultra-Weak Photon Emission from Human Hands Varies Diurnally. Biophotonics 2007: Optics in Life Science, 6633, Article ID: 66331J. https://doi.org/10.1117/12.727672

[31] Hari, S. (2010) Consciousness, Mind and Matter in Indian Philosophy. Journal of Consciousness Exploration \& Research, 1, 640-650.

[32] Van Wijk, R., et al. (2013) Imaging Human Spontaneous Photon Emission. Historic Development, Recent Data and Perspectives. Trends in Photochemistry \& Photobiology, 15, 27-40.

[33] Persinger, M.A. and Lavallee, C.F. (2010) Theoretical and Experimental Evidence of Macroscopic Entanglement between Human Brain Activity and Photon Emissions: Implications for Quantum Consciousness and Future Applications. Journal of Consciousness Exploration \& Research, 7, 785-807.

[34] Saroka, K.S., Dotta, B.T. and Persinger, M.A. (2013) Concurrent Photon Emission, Changes in Quantitative Brain Activity over the Proximal Geomagnetic Field While Imagining White Light. International Journal of Life Science and Medical Research, 3, 30-34. https://doi.org/10.5963/LSMR0301005

[35] Costa, J.N., et al. (2016) Differential Spontaneous Photon Emissions from Cerebral Hemispheres of Fixed Human Brains: Asymmetric Coupling to Geomagnetic Activity and Potentials for Examining Post-Mortem Intrinsic Photon Information. Neuroscience \& Medicine, 7, 49-59. https://doi.org/10.4236/nm.2016.72006

[36] Van Wijk, R., et al. (2006) Anatomic Characterization of Human Ultra-Weak Photon Emission with a Moveable Photomultiplier and CCD Imaging. Journal of Photochemistry and Photobiology B: Biology, 83, 69-76.

https://doi.org/10.1016/j.jphotobiol.2005.12.005

[37] Bordoni, B., et al. (2017) Emission of Biophotons and Adjustable Sounds by the Fascial System: Review and Reflections for Manual Therapy. Journal of Evidence-Based Integrative Medicine, 23, 1-6. https://doi.org/10.1177/2515690X17750750

[38] Van Wijk, E.P.A. and Van Wijk, R. (2005) Multi-Site Recording and Spectral Analysis of Spontaneous Photon Emission from Human Body. Forsch Komplementärmed Klass Naturheilkd, 12, 96-106. https://doi.org/10.1159/000083935

[39] Yang, J.M., et al. (2004) Biophoton Emission and Blood Flow in the Human Hand. Journal of International Society of Life Information Science, 22, 344-348.

[40] Voeikov, V.L., et al. (2003) Biophotons Research in Blond Reveals Its Holistic Properties. Indian Journal of Experimental Biology, 43, 473-482.

[41] Van Wijk E.P., Van Wijk, R. and Bajpai, R.P. (2008) Quantum Squeezed State Analysis of Spontaneous Ultra Weak Light Photon Emission of Practitioners of Meditation and Control Subjects. Indian Journal of Experimental Biology, 46, 345-352.

[42] Cifra, M., et al. (2007) Spontaneous Ultra-Weak Photon Emission from Human Hands Is Time Dependent. Radioengineering, 16, 15-19.

[43] Jung, H.H., et al. (2005) Year-Long Biophoton Measurements: Normalized Frequency Count Analysis and Seasonal Dependency. Journal of Photochemistry and Photobiology B: Biology, 78,149-154.

https://doi.org/10.1016/j.jphotobiol.2004.08.002

[44] Nakamura, K. and Hiramatsu, M. (2005) Ultra-Weak Photon Emission from Human Hand: Influence of Temperature and Oxygen Concentration on Emission. 
Journal of Photochemistry and Photobiology B: Biology, 80,156-160.

https://doi.org/10.1016/j.jphotobiol.2005.02.005

[45] Ho, M.W. (1995) Bioenergetics and the Coherence of Organisms. Neural Network World, 5, 733-750.

[46] Stohs, S.J. and Hartman, M.J. (2015) Review of the Safety and Efficacy of Moringa oleifera. Phytotherapy Research, 29, 796-804. https://doi.org/10.1002/ptr.5325 